\title{
The channel capacity of visual awareness divided among multiple moving objects
}

\author{
Joseph S. Lappin ${ }^{1,2,3}$ • Douglas L. Morse ${ }^{1,4}$ - Adriane E. Seiffert ${ }^{1}$
}

Published online: 29 June 2016

(C) The Psychonomic Society, Inc. 2016

\begin{abstract}
If attention is distributed among multiple moving objects, how does this divided attention affect the temporal process for detecting a specific target motion? Well-trained observers in three experiments monitored ongoing random motions of multiple objects, trying to rapidly detect nonrandom target motions. Response time hazard rates revealed a simple lawful structure of the detection processes. Target detection rates (hazard rates, in bits/s) were inversely proportional to the number of observed objects. Detection rates at any response time and in any condition equaled a product of two parallel (functionally independent and concurrent) visual processes: visual awareness and motion integration. The rate of visual awareness was inversely proportional to Set Size ( $n$ $=1-12$ ), constant over time, and invariant with integrated motion information. Thus, a single rate parameter, indicating a constant channel capacity of visual awareness, described detection rates over a wide range of conditions and response times. During an initial interval of roughly $0.5 \mathrm{~s}$, detection rates increased proportionally with the duration and length of motion; but after this initial integration, detection rates were constant, independent of the time the target remained undetected. The relationship between the quantity of visual information and detection rates was simpler than anticipated by
\end{abstract}

Joseph S. Lappin

joe.lappin@vanderbilt.edu

Vanderbilt University, Nashville, TN, USA

2 Discerning Technologies LLC, San Diego, CA, USA

39 Cubs Drive, Burnsville, NC 28714, USA

4 IKRG Inc., Nashville, TN, USA contemporary theories of attention, perception, and performance.

Keywords Visual awareness · Attention · Motion perception · Target detection · Visual search · Hazard rates . Response time $\cdot$ Capacity $\cdot$ Parallel processes

\section{Introduction}

Human attention is often spread over multiple streams of information - multiple objects and events in overlapping temporal patterns. Natural scenarios often demand attention to multiple tasks. When attention is divided this way, perception may be slower and observed objects less well resolved (Broadbent, 1958; Cherry, 1953; Eriksen \& Johnson, 1964). Such results have indicated that attention has "limited capacity."

Two general questions motivated the present study: If visual attention and perception have limited capacity, then how is this capacity distributed over multiple moving objects? How does the spread of attention affect temporal processes for detecting a target motion? These questions arose in a project to identify risks of information loss by human observers when multiple pieces of salient information compete for attention.

We investigated dynamic scenarios with (a) multiple moving objects, (b) no involvement of visual memory, and (c) continuous observation intervals in which target information occurs unpredictably. These task conditions distinguished the present study from many other laboratory studies of related issues, but these conditions are not ecologically unusual. Experiments with discrete trials and predictable and distinct onsets add non-visual information about stimulus onset times. In contrast, visual target information here depended entirely 
on perceived differences between the target and background motion paths.

Target motions in this task were indistinguishable from the background at any single time or place. The target trajectory was an approximately linear path toward a distinct moving "prey" object. Before onset of the target motion, all of the displayed objects moved randomly; any one could initiate a target motion. Target motions occurred at multiple, unpredictable random times during a continuous observation period of several minutes. The main independent variable was the Set Size $(n)$ of randomly moving objects. The dependent variable was the speed of the observer's detection responses.

Mean response times (RTs) increased linearly with Set Size, as shown in Fig. 2a. This result is easily recognized as a common characteristic of visual search (see Eckstein, 2011; Logan, 2004; Wolfe \& Horowitz, 2004). A linear relation between Set Size and mean RT is not surprising, although a nonlinear effect might have been predicted: A limited span of apprehension (e.g., Fisher, 1984; Luck \& Vogel, 1997; Miller, 1956; Pylyshyn \& Storm, 1988) might encompass a small set of objects (e.g., $n=2$ ) while larger sets (e.g., $n=6$ or 8 ) might not be perceived simultaneously, requiring serial search. The obtained Set Size effect did not support this limited span hypothesis, but the linear relation was not surprising.

One might wonder, however, whether the RT distributions offer a fuller picture of the temporal processes. Indeed, the RTs were highly variable, with standard deviations for each observer in each condition larger than the effect of Set Size on mean RT. Accordingly, we began exploratory analyses of the RT distributions, with special interest in the hazard functions. (The nature and rationale of hazard rate analyses are described below.) Surprisingly, the hazard rates revealed an unexpectedly simple structure of the visual processes. That finding is the focus of this report.

This study was not designed to test specific hypotheses. Instead, we sought to clarify visual capacity limitations in perceiving changing patterns of multiple moving objects. The Set Size of observed objects determined both the quantity of spatiotemporal information and the observer's uncertainty about which object might become a target for detection.

\section{Overview of experiments}

The principal independent variable was the number of randomly moving objects the observer had to monitor to detect a non-random target motion. Across experiments, Set Size, $n$, varied from 1-12 objects. The principal dependent variable was the RT for detecting a target motion. Rather than the usual parameters for RT distributions, however, the detection process was revealed more clearly by the response time hazard rates.
Experiment 1 varied only the Set Size $(n=2,4,6,8)$ of moving objects monitored by the observers. Experiment 2 tested the generality of the results by investigating the effects of Motion Speed and the effects of Set Sizes of $n=1$ and 12 as well as 4. Experiment 3 tested the visual role of a distinct "prey" object by comparing performance both with and without this visible target destination.

\section{General method}

Displays of multiple moving objects Visual information consisted of multiple randomly moving objects. Prior to the onset of a target motion (described below), display information and target uncertainty were proportional to the number of moving objects.

The objects were high-contrast solid black circles, 3.53$\mathrm{mm}$ diameter, and 17 arcmin at a viewing distance of $72 \mathrm{~cm}$. These objects $\left(1.4 \mathrm{~cd} / \mathrm{m}^{2}\right)$ appeared on a medium grey background $\left(102 \mathrm{~cd} / \mathrm{m}^{2}\right)$. A Set Size of $n=\{1,2,4,6,8,12\}$ such objects was randomly distributed within a $26.5 \times 26.5 \mathrm{~cm}$ $\left(20.1 \times 20.1^{\circ}\right.$ visual angle $)$ area marked by visible boundary lines. To reduce local clusters, each dot was initially positioned near the center of a randomly selected cell in a $5 \times 5$ imaginary grid, with only one dot per cell. Visual fixation was unconstrained, and there was no fixation point. Observers controlled viewing distance by aligning head position with the table supporting the video monitor (ViewSonic VX924, $60 \mathrm{~Hz}, 19$ in., 1,280 × 1,024 pixels). Displays and timing were controlled by a Mac Mini computer and by Matlab and PsychToolbox software.

Prior to the onset of a non-random target motion, each of the objects moved independently in randomly changing directions. The probability of a direction change was 0.5 in each successive frame. A new direction was given by a vector sum of the previous direction plus a random direction uniformly distributed in the interval $36-324^{\circ}$, weighted by a "wiggle" parameter. (The formula for direction change was NewDirection $=$ OldDirection $+w \cdot$ rand , where rand $=$ $\left\{36^{\circ}, 72^{\circ}, 108^{\circ}, \ldots 324^{\circ}\right\}$, and the wiggle parameter, $w$, was $w=0.2$.) In Experiments 1 and 3, the random motion speed was 2 pixels $/$ frame $=2.79 \%$; and in Experiment 2, the speed was varied $-1,2$, or 3 pixels $/$ frame $=1.40,2.79$, or $4.19^{\circ} / \mathrm{s}$.

Moving target detection task The observers' task was to rapidly detect a salient target motion directed in "pursuit" toward a visibly designated "prey" object. The "prey" was a visibly distinct bright red circle $\left(53 \mathrm{~cd} / \mathrm{m}^{2}\right)$ of the same size as the other moving objects. The prey object also moved, though slowly and with less random direction change than the other randomly moving objects. The speed of the prey object was $1.40 \%$ in all three experiments, and the random "wiggle" parameter for the prey was $w=0.03$. The prey object was 
clearly visible throughout the ongoing observation period. Observers reported using the prey as a reference point for the motions of other objects, to aid detection of a target motion toward the prey. The trajectory of target motion was approximately linear and directly toward the position of the red prey object, changing in direction with the moving prey, with increasing directional change as the pursuer was closer to the prey. Figure 1 illustrates this target detection task.

Target (pursuit) motions occurred unpredictably, with a constant probability in each $60 \mathrm{~Hz}$ frame, unless (a) an undetected target motion was already underway, (b) a detection response (including false alarms) had occurred within the preceding $0.2 \mathrm{~s}$, or (c) the pursuit object was within 96 pixels $(=8$ object diameters) of the prey. Time between successive target motions was highly variable (exponentially distributed); and the number of targets within a given 3 min observation period was also variable, averaging 18 targets per min.

A detection response terminated the pursuit motion, causing resumption of the object's random motion. If the observer failed to detect the pursuit before it was within 1.5 object diameters (18 pixels) of the red prey, then this was recorded as a missed target motion. Over all conditions and observers in Experiment 1, $0.9 \%$ of the target motions were missed, ranging from $0.1 \%$ with set size 2 to $1.7 \%$ with set size 8 . If the observer failed to detect the target motion before it reached the prey, then the pursuing object jumped immediately to its original position at the start of the 3- or 4-min observation period. This event was quite visible, and gave immediate feedback that the target had been missed.

Performance was evaluated mainly by RTs for correct detections (hits), measured from the onset of target motion to depression of the keyboard spacebar. Frequencies of hits, misses, and false alarms were also evaluated. Responses when there was no target motion were recorded as false alarms, though RTs for the false alarms were undefined. RTs in the interval 200-300 ms were also counted as false alarms. (RTs $<200 \mathrm{~ms}$ were removed from analysis, in order to eliminate inadvertent response chains caused by observers failing to release the spacebar or pressing it repeatedly.) Also, with no explicit 'NO' response, frequencies of "correct rejections" were undefined. A choice task would have provided an accuracy measure; but pilot work found that multiple alternative responses to multiple alternative targets (two sets of pursuit objects with different colors) yielded very unreliable RTs and accuracies. A primary aim was to measure detection speed, so the present single-response task was found to be best for our purposes.

Procedures Observers practiced for at least three sessions, until their response times were not decreasing. All observers understood the purpose of the experiments.

Observers responded by pressing the spacebar on the computer keyboard as quickly as possible to the onset of a target motion. The objective was to minimize RTs, numbers of missed detections, and false alarms. The observers understood that the aim was to estimate reliable and valid measures of detection times, and that occasional misses and false alarms were inevitable but should be very infrequent relative to the hits. Misses and false alarms varied slightly between conditions and observers, but the magnitude and variations of these errors were insufficient to alter interpretation of the detection RTs.

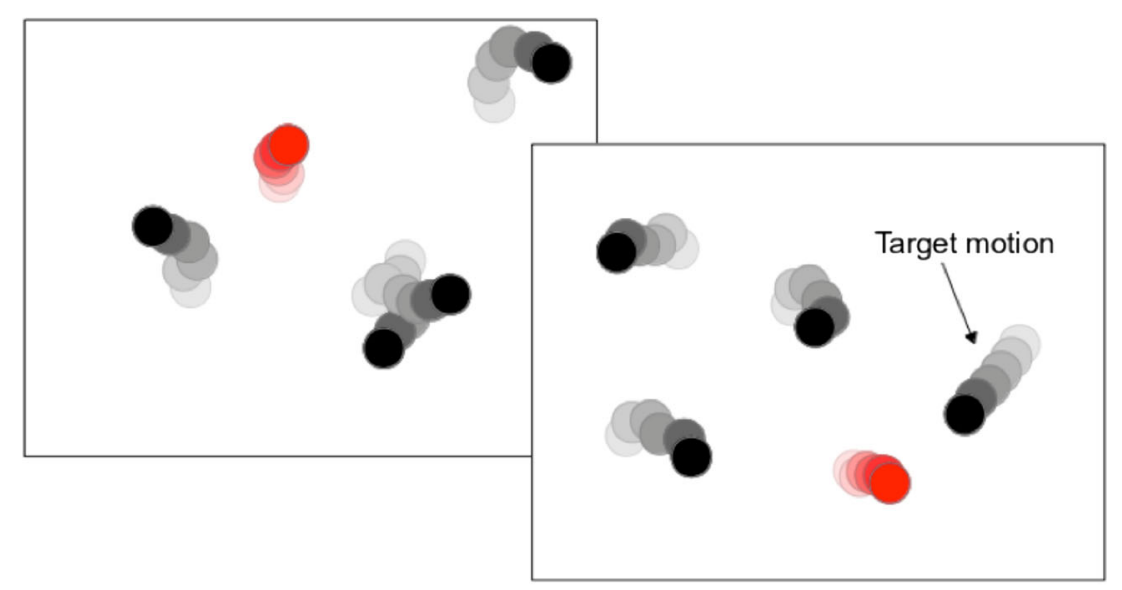

Time

Fig. 1 Schematic illustration of the target motion detection task. Prior to a target motion, all the black objects moved randomly and independently. At unpredictable random times during a continuous observation interval, the motion directions of one object changed from random to "pursuit" toward the red "prey" object. This prey object was also moving but (in most conditions) more slowly and with less direction change than the other moving objects. The Set Size $(n=1-12)$ of randomly moving objects was an independent variable; and the dependent variable was the RT for detecting the non-random target motion 
Experiment 1 involved 12 sessions, each consisting of four 3-min observation periods, one for each of the four set size (n $=2,4,6,8)$ conditions. Each observer started the computer program for that session. There was a brief break between each observation period while the observer recorded on paper the average RT and numbers of false alarms and misses for that condition. The observer then initiated the next observation period when he or she was ready. The computer randomized the order of the four set size conditions within each session. Each such session required about 20-25 min. Observers often ran two sessions during the same visit to the laboratory, typically with at least a 5-min break between sessions. The number of target motions in each observation period was variable, averaging 55 target motions. Thus, the number of target motions for each observer in each set size condition averaged about 663 trials for the 12 sessions. The mean RT for each session, observer, and condition was sufficiently stable to justify pooling data across sessions for each condition and observer.

Procedures for Experiments 2 and 3 were essentially the same. Changes in procedures are noted below for those experiments.

Observers The observers included the authors, a postdoctoral researcher at Vanderbilt with previous experience in studies of motion perception, and an undergraduate research assistant. The age range was from approximately 20-73 years. Performance was similar across observers; though the two youngest observers tended to be slightly faster. Three were male and two were female. All had normal or corrected-to-normal acuities. All were fully informed about the nature and purposes of the experiments.

Five observers served in Experiment 1, four in Experiment 2, and three in Experiment 3. JL, DM, and KD served in all experiments, AS in Experiments 1 and 2, and $\mathrm{BD}$ in Experiment 1.

Hazard rates Our initial analyses relied on the mean RT. The RT distributions for each observer in each condition were highly variable, however, and this motivated additional analyses of temporal structure the RT distributions. Specifically, hazard functions were found to provide a detailed and clear picture of the temporal process.

The hazard rate at any time $t$ is based on the rate of change in the survival function of not-yet-detected targets. The present analysis was adapted from previous work especially by Townsend and colleagues (e.g., Godwin et al., 2015; Houpt \& Townsend, 2012; Townsend, 1990; Townsend \& Ashby, 1983; Townsend \& Eidels, 2011; Townsend \& Wenger, 2004b; Wenger \& Gibson, 2004).
If $f(t)$ is a probability density, and $F(t)$ is the cumulative distribution function (CDF), then the survival function is $S(t)$ $=1-F(t)$, and the hazard rate, $h(t)$, is

$h(t)=f(t) / S(t)$.

The integrated hazard function, $H(t)$, is useful and easily computed: ${ }^{1}$

$H(t)=\int_{0}^{t} h\left(t^{\prime}\right) d t^{\prime}=-\log S(t)$.

This integrated function, $H(t)$, is simply a logarithmic transform of the cumulative distribution function, $F(t)$, preserving the ordinal structure of the RT distribution.

The base of the logarithm is arbitrary, affecting only the numerical scale. The natural $\log , \ln (x)$, is conventional in the literature, but for heuristic reasons and terminology, we adopted $\log _{2}: H(t)=-\log _{2} S(t){ }^{2}$ Thus, the units of $H(t)$ are given here in bits. The median RT is the time $t$ at which $H(t)$ $=1.0 ; H(t)=2.0$ is at the $25^{\text {th }}$ percentile of the survivor function, and so forth. The hazard rate, $h(t)$, is the temporal derivative of $H(t)$, with units of bits/s. The reciprocal, $1 / h(t)$, in $\mathrm{s} /$ bit, is the half-life of targets not yet detected at time $t$.

Analyses of RT data often focus on probability distributions as the principal objects of interest, but the hazard functions can provide more direct information about the temporal processes (Bloxom, 1984; Godwin et al., 2015; Luce, 1986; Townsend, 1990; Townsend \& Eidels, 2011; Townsend \& Nozawa, 1995; Wenger \& Gibson, 2004). Importantly, the form of a hazard function can be invariant with substantial changes in the RT mean and variance. The present results illustrate that fact.

Hazard functions, both $h(t)$ and $H(t)$, are ratio-scaled measures of detection rates at a given point in time. Hazard rates, $h(t)$, are based on the differential structure rather than the RT probability distributions as such (see Townsend, 1992). Hazard functions are non-parametric descriptions requiring no assumptions about the RT probability distributions or the underlying stochastic process. These ratio-scaled measures were essential to the findings of this study.

\footnotetext{
${ }^{1}$ This formula exemplifies an elementary integration formula: $\int f(t) /(1-$ $F(t)) d t=-\log (1-F(t))+C$. Here, $C=0$, since $F(0)=0$. As an illustration, imagine that the detection process involves randomly drawing balls from an urn, replacing the ball after each selection, and continuing until a target ball is found. If $p$ is the probability of detecting the target ball on each draw, and if $P(N)$ is the probability of finding the target ball within $N$ draws or fewer, then the probability of not finding the target within $N$ draws, the survival function, is $1-P(N)=(1-p)^{N}$. Thus, $\log (1-P(N))=N$ $\log (1-p)$.

${ }^{2} \log _{2}(x)=\ln (x) / \ln (2)=(1.4427) \ln (x)=\log _{10}(x) / \log _{10}(2)=(3.3219)$ $\log _{10}(x)$.
} 
The momentary hazard rate, $h(t)$, measures the slope or rate of change in $H(t)$ at time $t$; and it is not determined by the value of $H(t)$ at that time. $H(t)$ is scaled in bits, and $h(t)$ is scaled in bits/s. Functional differences between the integrated and momentary hazard functions, $H(t)$ and $h(t)$, are examined in Appendix 1.

Unfortunately, estimates of momentary hazard rates are often unreliable, with local irregularities amplified especially as response frequencies diminish toward the lower tail of the survivor function (see Cechile, 2003; Luce, 1986; Houpt \& Townsend, 2012; Van Zandt, 2000). The integrated, $H(t)$, functions can bypass aspects of this estimation problem (see Houpt \& Townsend, 2012; Wenger \& Gibson, 2004). Relative values of hazard functions for two conditions can be quantified by differences in their $\log H(t)$ values. Wenger and Gibson (2004) provide an empirical demonstration of the power of this method; and Houpt and Townsend (2012) develop basic statistical analyses. Comparisons of the $\log H(t)$ functions were also used to evaluate the effects of Set Size in Experiment 1 of the present study, as shown in Appendix 1. Limitations of analyses of the integrated functions, $H(t)$, however, led us to focus instead on the momentary hazard rates, $h(t)$. The structure and variations of the temporal process were described more clearly in the momentary hazard rates, $h(t)$, than in the integrated functions, $H(t)$. Appendix 1 examines differences between these two analytic approaches, and justifies reliance on the momentary hazard rates.

Descriptive and statistical analyses of hazard rates in this study developed through exploratory analyses with several computational methods. ${ }^{3}$ Consistent results were obtained by estimating slopes of the integrated hazard functions at five quantiles - corresponding to $20 \%$ intervals of the CDF of RTs, where $F(t)=\{0.10,0.30,0.50,0.70,0.90\}$. Thus, the estimated momentary hazard rate was obtained from the difference in values of the integrated function $H(t)$ relative to the difference in RTs at two points where $F(t)$ and $S(t)$ changed by $20 \%$. Specifically, the estimated hazard rate at time $t$ was $h(t)$ $=[H(t+\Delta t)-H(t)] / \Delta t$, where $t$ and $t+\Delta t$ were the RTs at successive $20 \%$ differences in the distribution function $F(t)$ $-10-30 \%, 30-50 \%, 50-70 \%$, and $70-90 \%$. The values of

\footnotetext{
${ }^{3}$ Exploratory analyses compared the effects of Set Size at the same time bins and at the same CDF quantiles for different conditions. Analyses with the same time bins for different conditions were all hampered by large differences in response frequencies for different Set Size conditions in specified time bins. When response frequencies were small, estimated hazard rates were unreliable. Estimating the slopes of the integrated $H(t)$ functions at specific quantiles of the CDF yielded the clearest comparisons across Set Size conditions. These quantiles also preserved information about the medians and spreads of the distributions.

Choosing the intervals between successive quantiles involves a tradeoff between the number of data points and the number of responses in each quantile interval - i.e., between temporal resolution and reliability of the local estimates. For the present data, the most consistent results seemed to be obtained with $20 \%$ quantile differences. This ad hoc choice was based on the present data, and might not generalize to other data.
}

$H(t)$ and $H(t+\Delta t)$ were specified and equal across conditions, but the $t$ and $\Delta t$ values were empirical and differed between conditions. Evaluating $H(t)$ at five quantiles yielded four independent estimates of the local slopes and hazard rates, $h(t){ }^{4}$

\section{Experiment 1}

The purpose was to evaluate the effect of Set Size on target detection speed. The independent variable was Set Size, $n=$ $\{2,4,6,8\}$.

Prior to onset of the target motion, the task demanded attention to the entire set of $n$ moving objects, although no overt behavioral response was needed. Perception was measured by the speeds with which observers detected a target motion by one of the objects - a change from random to non-random pursuit toward the moving prey, which occurred at unpredictable times. These two aspects of visual information - the multi-object random motion background and the nonrandom individual target motion - jointly influenced the detection responses.

\section{Results and discussion of Experiment 1}

Analyses of these RT data developed through exploratory analyses. The principal results were discovered, not predicted or anticipated when data analyses began. The hazard rates turned out to be essential.

Mean RTs and relative frequencies of hits, misses, and false alarms were examined for each session, and were found to be consistent across sessions. Accordingly, these data were combined across the 12 sessions for each observer in each Set Size condition.

Data for individual observers were qualitatively and quantitatively similar. For simplicity, most of the following graphs describe responses combined over all observers, as if from a single observer. The data for individual observers were also examined numerically, graphically, and statistically. Individual observers differed only in quantitative details, with no apparent significant qualitative differences. Naturally, the spreads of combined distributions were slightly greater than those for individual observers, but this increased spread was small. The combined distributions were more stable and were quite representative of the individual results. Descriptive models and statistical tests were applied to the data of individual observers as well as the combined data.

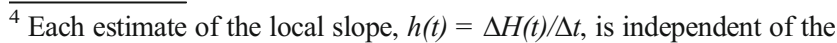
estimates at other points because each is computed from a different set of responses. The estimated hazard rate, or slope, is determined by the denominator, $\Delta t-$ by the duration containing the next $20 \%$ of the responses.
} 
Means and standard deviations Figure 2a shows the mean RTs and standard deviations (SDs) of the detection hits for each observer in each condition. The error bars are SDs, not standard errors. With an average of 663 responses for each of the displayed means, standard errors were roughly $4 \%$ of these SDs. Thus, the effect of Set Size was highly significant, for each of the individual observers as well as across observers. The linear increase of mean RT with Set Size was $620+60 \mathrm{~ms} /$ item $(r=0.998)$. The average $F$-ratio for the five individual observers was $F\left(3 / d f_{\text {ave }}\right)=162.2$, where the average $d f_{a v e}=2622$. As may be seen, the rank-orders of mean RTs for the four Set Size conditions were the same for each of the five observers.

The linear relation between Set Size and mean RT, however, is an insufficient description of the detection process. First, the SDs were large. Detection times were quite variable from one trial to the next, even for the same observer and same condition. The SDs averaged $31 \%$ of the mean RT; and the slope of the increase in SD with Set Size was similar to that for the mean RTs: $58+47 \mathrm{~ms} /$ item (based on the RMS SDs for each observer and condition). This variability was partly attributable to the displayed information, with random spatial patterns and motions, but it also reflects variability of the detection processes.

Second, because the spread of the RT distributions increased with Set Size, the slope of the relation between Set Size and RT depends on the detection probability criterion (a quantile of the CDF of RTs). Figure $2 b$ illustrates the functional importance of the probability-ofdetection criterion. Detection times/item increased more than fivefold for a $90 \%$ detection criterion as compared to a $10 \%$ detection criterion. (These regression lines were computed on the combined RT distributions of all observers.)

Table 1 gives the frequencies of hits, misses, and false alarms, and the mean RT for hits for each observer in each Set Size condition. Misses were infrequent, occurring for $1 \%$ of the target motions. False alarms accounted for 2.5 $3 \%$ of the overt detection responses, and were slightly more frequent in the Set Size 2 condition with the fastest detection responses.

Hazard rates Effects of Set Size on the RT distributions may be described by integrated hazard functions, $H(t)=-$ $\log _{2}(S(t))$. These functions are shown in Fig. 3a. The unit of measure on the vertical axis is bits, where 1 bit corresponds to a $50 \%$ reduction in the number of undetected target motions; and the median RT is the value of $t$ where $H(t)=1.0$. The integrated hazard functions in Fig. 3a were computed from the combined responses of all observers. The data points correspond to the 90th, 70th, 50th, 30th, and 10th percentiles of the survivor functions, $S(t)$, for the combined responses from all observers.
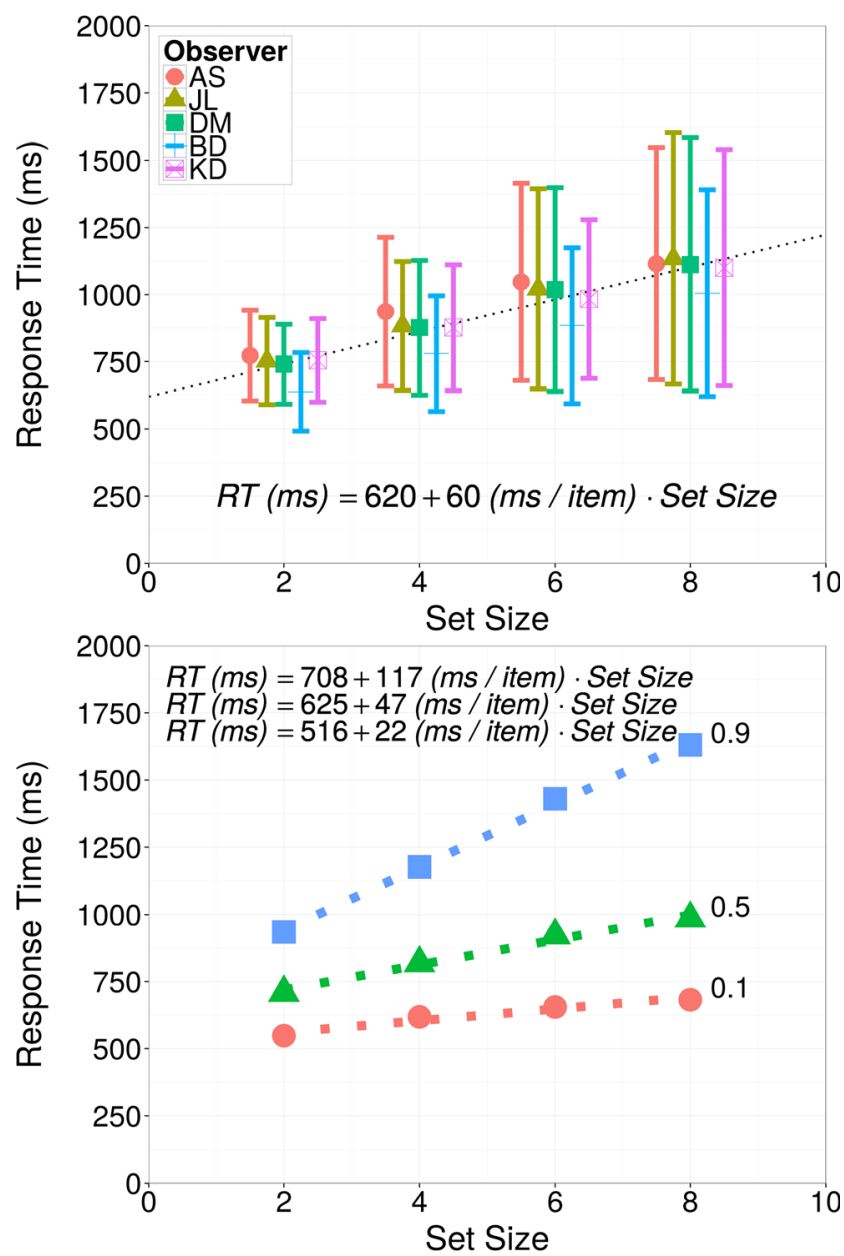

Fig. 2 a Means and standard deviations for each observer in each Set Size condition in Experiment 1. (Error bars are SDs, not standard errors. The number of responses for each data point averaged 663.) b Set Size effects for three values of detection probability, $F(t)$

Salient features of these integrated functions are their slopes - which decreased as $n$ increased. These integrated functions were roughly linear, but initially accelerated during the fastest RTs, at the lower left of the curve. The slopes of the nearly linear segments of these functions are approximately proportional to $1 / n$. Thus, most of the data in Fig. 3a are consistent with a single parameter!

Slopes of the $H_{n}(t)$ integrated functions correspond to hazard rates, $h_{n}(t)$, in bits/s. These hazard rates, $h_{n}(t)$, were estimated by the ratios of differences in the vertical and horizontal axes of $H(t): h\left(t_{i}\right)=\left[H\left(t_{i+1}\right)-H\left(t_{i}\right)\right] /\left[t_{i+1}-t_{i}\right]$, where the index $i$ $=\{1,2,3,4\}$ denotes the first four points from the left on the integrated functions $H(t)$ (at the 10th, 30th, 50th, and 70th percentiles of the CDF).

Figure $3 \mathrm{~b}$ shows detection hazard rates as a function of time from onset of the target motion for each Set Size condition, based on the combined RTs of all observers. The hazard rates plotted in this graph have been multiplied by 
Table 1 Frequencies of hits, misses, false alarms (FAs) and mean response time (RT) (ms) for each observer and Set Size in Experiment 1

\begin{tabular}{|c|c|c|c|c|c|c|}
\hline Observer & 2 & 4 & 6 & 8 & Totals & Hit rate \\
\hline \multicolumn{7}{|l|}{$A S$} \\
\hline Hits & 608 & 652 & 653 & 635 & 2,548 & 0.993 \\
\hline Misses & 0 & 5 & 6 & 8 & 19 & \\
\hline FAs & 20 & 11 & 15 & 15 & 6 & \\
\hline Mean RT & 772 & 936 & 1,047 & 1,115 & & \\
\hline \multicolumn{7}{|l|}{$J L$} \\
\hline Hits & 661 & 690 & 742 & 692 & 2785 & 0.983 \\
\hline Misses & 2 & 15 & 9 & 21 & 47 & \\
\hline FAs & 34 & 24 & 20 & 30 & 108 & \\
\hline Mean RT & 752 & 883 & 1,022 & 1,134 & & \\
\hline \multicolumn{7}{|l|}{$D M$} \\
\hline Hits & 665 & 701 & 698 & 692 & 2,756 & 0.990 \\
\hline Misses & 1 & 2 & 12 & 13 & 28 & \\
\hline FAs & 18 & 25 & 19 & 28 & 90 & \\
\hline Mean RT & 740 & 876 & 1,019 & 1,112 & & \\
\hline \multicolumn{7}{|l|}{$B D$} \\
\hline Hits & 636 & 626 & 615 & 609 & 2,486 & 0.992 \\
\hline Misses & 1 & 3 & 10 & 7 & 21 & \\
\hline FAs & 22 & 14 & 20 & 6 & 62 & \\
\hline Mean RT & 638 & 780 & 884 & 1,005 & & \\
\hline \multicolumn{7}{|l|}{$K D$} \\
\hline Hits & 611 & 662 & 653 & 627 & 2,553 & 0.995 \\
\hline Misses & 0 & 1 & 4 & 7 & 12 & \\
\hline FAs & 5 & 13 & 12 & 5 & 35 & \\
\hline Mean RT & 754 & 877 & 983 & 1,100 & & \\
\hline Total & 2 & 4 & 6 & 8 & & \\
\hline Hits & 3,181 & 3,331 & 3,361 & 3,255 & 13,128 & 0.990 \\
\hline Misses & 4 & 26 & 41 & 56 & 127 & \\
\hline FAs & 99 & 87 & 86 & 84 & 356 & \\
\hline Mean RT & 731 & 871 & 991 & 1,093 & & \\
\hline Hit Rate & 0.999 & 0.992 & 0.988 & 0.983 & 0.990 & \\
\hline $\mathrm{FA} /(\mathrm{H}+\mathrm{FA})$ & 0.030 & 0.025 & 0.025 & 0.025 & 0.026 & \\
\hline
\end{tabular}

the Set Size, $n \bullet h(t)$, to show the common temporal structure of the hazard functions for all four Set Size conditions. As may be seen, the obtained hazard rates are closely approximated by two straight lines: (1) For $c \leq t \leq k, n \bullet h_{n}(t)=a \bullet(t-$ $c) /(k-c)$; and (2) for $\mathrm{t}>\mathrm{k}, n \bullet h_{n}(t)=a$. Thus, three parameters describe the 16 data points: $c=0.42 \mathrm{~s}, k=0.86 \mathrm{~s}$, and $a=27.9 \mathrm{bits} / \mathrm{s}$.

The parameters were estimated by minimizing standardized squared deviations between obtained and predicted hazard rates: $\left(h_{n}(t)-P_{n}(t)\right)^{2} / P_{n}(t)$, where $h_{n}(t)$ and $P_{n}(t)$ are the obtained
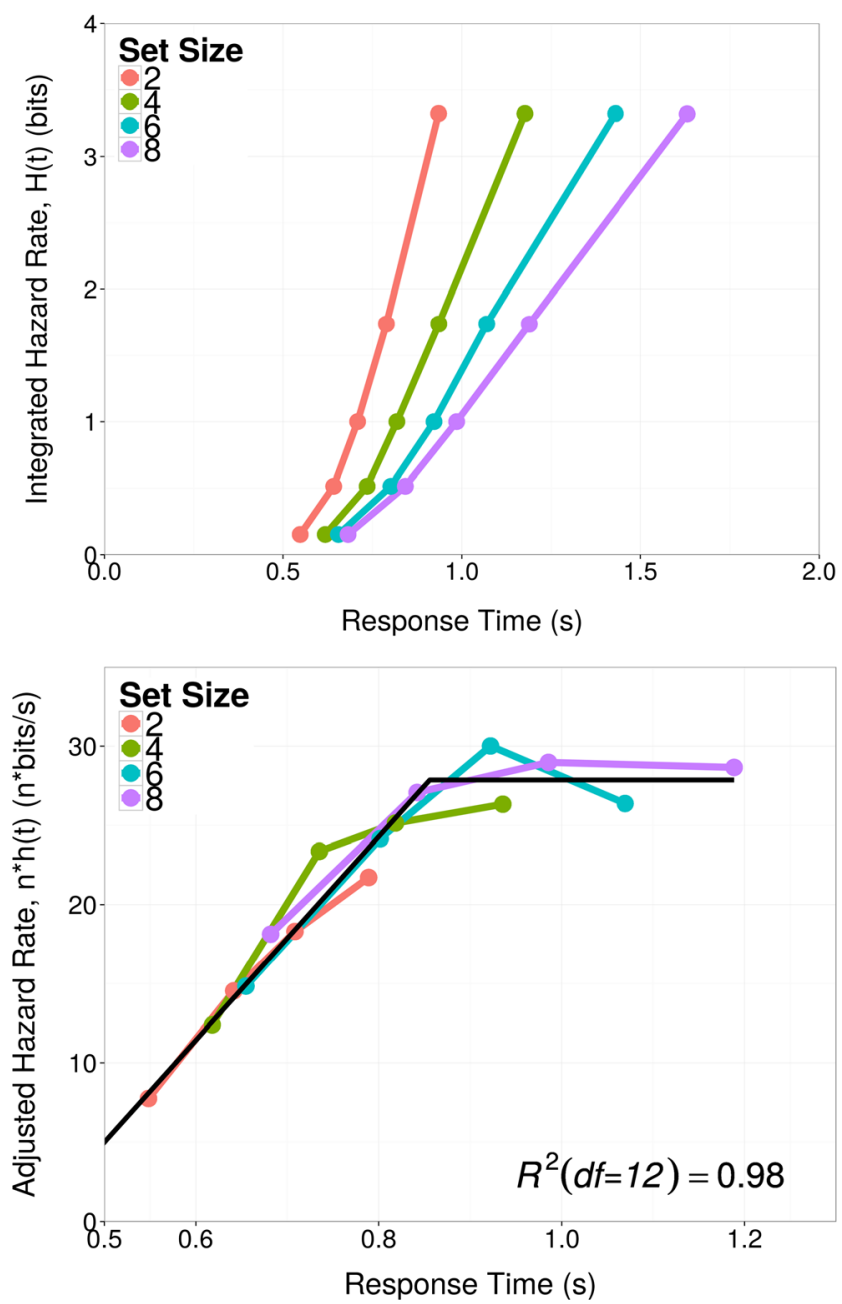

Fig. 3 a Integrated hazard functions for each Set Size in Experiment 1. Data points correspond to the 90th, 70th, 50th, 30th, and 10th percentiles of the survivor functions for the pooled data of all five observers. The measure on the vertical axis is $H(t)=-\log _{2} S(t)$, where $S(t)$ is the survivor function. b Hazard rates for the data in a. Each data point is the slope (bits/s) of the line segment between successive pairs of points on $H(t)$. Three parameters specify the descriptive model given by the two solid black lines: for $c \leq t \leq k, n \bullet h_{n}(t)=a \bullet(t-c) /(k-c)$; and for $t>k, n \bullet h_{n}(t)=a$; where $a=27.9$ bits $/ \mathrm{s}, c=0.42 \mathrm{~s}$, and $k=0.86 \mathrm{~s}$

and predicted values of the hazard rates. ${ }^{5}$ These three-parameter equations account for $98 \%$ of the variance of the 16 data points $(d f=12)$. This descriptive model was suggested by the

\footnotetext{
${ }^{5}$ This chi-square-like standardized measure scaled deviations relative to the predicted scores, which varied substantially with both Set Size, and time. Ordinary least-squares deviations depended mainly on the small Set Size conditions, which had higher hazard rates and relatively larger deviations from predictions. Ordinary least-squared deviations assume interval-scaled data, for which ratios are not meaningful. The hazard rates, however, are ratio scales, for which ratios such as $(O-P)^{2} / P$ are meaningful. The degrees of freedom for the sums of squared deviations was $N$ $1-Q$, where $N$ is the number of data points and $Q$ is the number of parameters. The total sums of squares was similarly scaled relative to the mean: $\sum(X-M)^{2} / M$, with $d f=N-1$. Thus, adjusted $R^{2}=1-$ $\left(\mathrm{SS}_{\text {predicted }} /(N-1-Q)\right) /\left(\mathrm{SS}_{\text {total }} /(N-1)\right)$.
} 

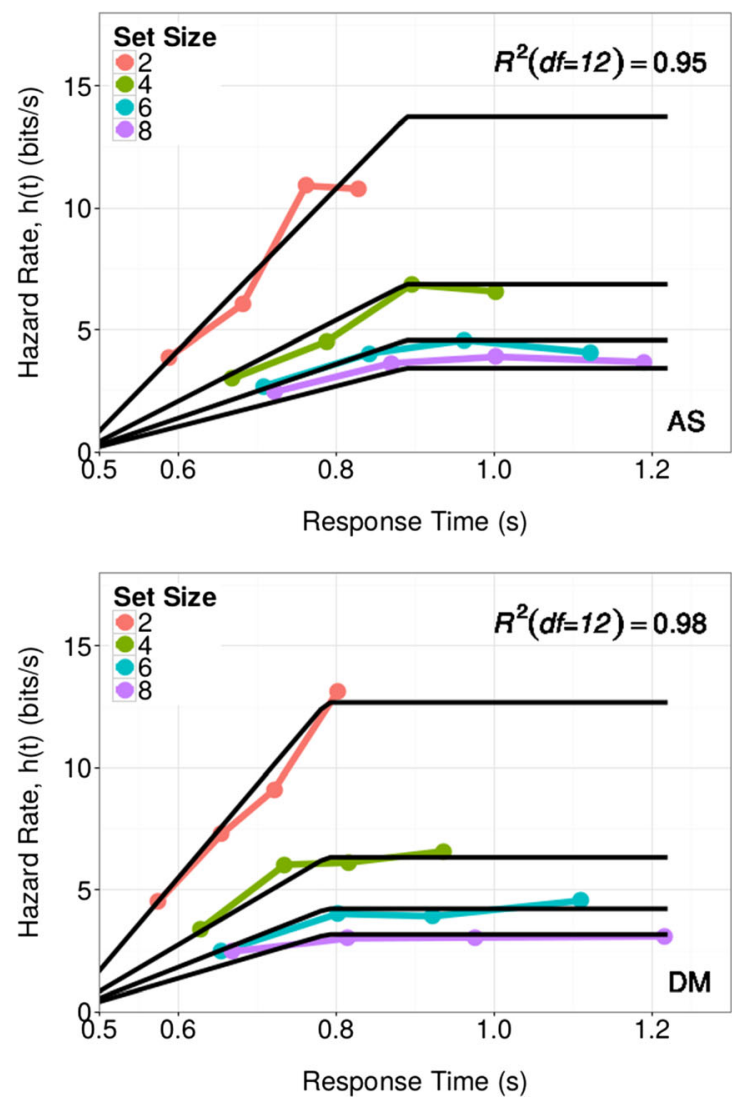

Fig. 4 Obtained and predicted detection hazard rates for four of the five observers in Experiment 1. Parameters of the predicted functions are given in Table 2. Parameters were estimated by minimizing

observation that slopes of the $H_{n}(t)$ functions were approximately proportional to $1 / n$. Parameter estimation and the evaluation of the model's goodness of fit were based on the obtained hazard rates, not multiplied by Set Size (unlike Fig. 3b).

This model was also fit to the 16 data points for each observer. Figure 4 shows obtained and predicted $h_{n}(t)$ values for four of the five observers. (Data for observer BD are not included in the graph merely for reasons of space. Also, BD

Table 2 Parameters of estimated hazard functions, $h_{n}(t)=A(n) \cdot M(t)$, where $A(n)=a / n, a$ is the detection rate in bits $/ \mathrm{s}, n$ is Set Size, $M(t)=(t-$ $c) /(k-c)$ for $c \leq t \leq k$, and $M(t)=0$ if $t<c, M(t)=1$ if $t>k$. Estimated parameter values are given for each observer and for the combined response times of all five observers in Experiment 1. The obtained and predicted data are shown in Figs. $3 \mathrm{~b}$ and 4

\begin{tabular}{lllll}
\hline Observer & $c(\mathrm{~s})$ & $k(\mathrm{~s})$ & $a$ (bits/s) & $R^{2}$ \\
\hline AS & 0.474 & 0.889 & 27.5 & 0.95 \\
JL & 0.448 & 0.848 & 27.3 & 0.89 \\
DM & 0.456 & 0.788 & 25.4 & 0.98 \\
BD & 0.379 & 0.704 & 30.3 & 0.97 \\
KD & 0.480 & 0.843 & 29.4 & 0.93 \\
Average & 0.447 & 0.814 & 28.0 & 0.95 \\
Combined & 0.423 & 0.856 & 27.9 & 0.98 \\
\hline
\end{tabular}
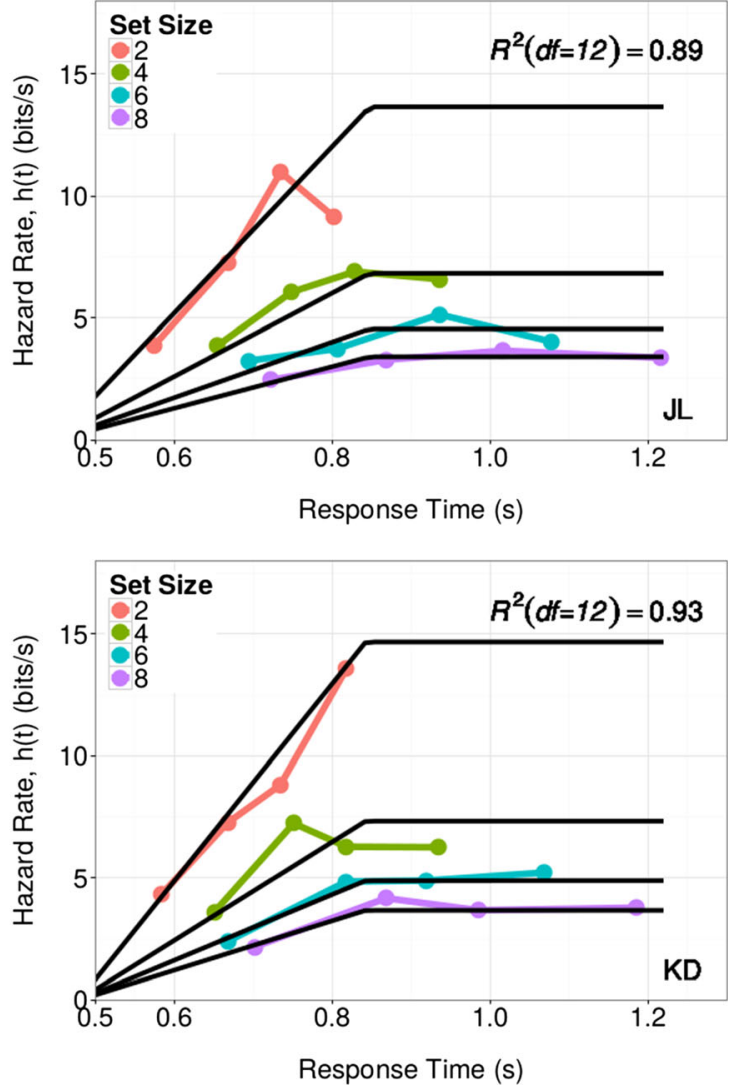

standardized squared deviations, $(y-P)^{2} / P$, where $y$ is the observed hazard rate and $P$ is the predicted value. Parameters are in Table 2

did not participate in Experiments 2 or 3.) Estimated parameters and $R^{2}(d f=12)$ measures of fit are given in Table 2 for each observer and for the combined data. The model parameters were similar across the five observers. Observer BD (youngest of the five observers) exhibited slightly faster time parameters, $c, k$, and $k-c$, and a higher asymptotic detection rate, $a$, than the other observers - effects also indicated by her faster mean RTs and smaller SDs (Fig. 2a).

The temporal structure described in Figs. $3 \mathrm{~b}$ and 4 and Table 2 indicates that the detection rate (hazard rate, not RT) at any given time was a product of two parallel (independent and concurrent) visual processes. These two functionally independent processes are identified by the observed selective influence of two variables, Set Size and time: The effects of varied Set Size were invariant with time; and the effects of time were invariant with Set Size. The mutual influence of both factors across the range of RT and hazard rates indicates that the two processes had concurrent influence. Thus, a wide range of observed hazard rates can be closely approximated as a product of two very simple and independent functions, each associated with a different visual process.

(1) Rate of visual awareness and detection: At any time throughout the temporal process, the hazard rate for 
target detection was proportional to $1 / n$. Across wide variations in response time and Set Size, detection rates were specified by a single rate parameter, $a$, such that $h_{n}(t) \propto a / n$. Evidently, the awareness rate parameter, $a$, corresponds to a constant capacity limit divided by the number of observed objects. Importantly, the hazard rates in Figures 3 and 4 were approximately constant after reaching an asymptote. Constant hazard rates identify an exponential probability distribution.

(2) Motion integration: Detection rates, however, did increase in proportion to elapsed time between about $0.45-0.81 \mathrm{~s}$, on average, as given by parameters $c$ and $k$. Linearly increasing detection rates proportional to the duration and distance of motion are compatible with the increasing optical and statistical differences between the target and random background motions. Visual integration of the motion paths would produce such a proportional relation between duration and target detection. Parameters $c$ and $k$ evidently mark the beginning and end of this visual integration. Parameter $c$ is a minimum RT, probably reflecting response processes. The difference $k-c$ estimates the duration of motion integration. Optical information about the target motion continues for a longer duration, so this temporal limit of visual integration is noteworthy. Importantly, the motion integration times were invariant with Set Size and mean RT.

Thus, the obtained detection rates reflect contributions of two functionally independent visual processes - one inversely proportional to Set Size and invariant with time, and the other invariant with Set Size and proportional to elapsed time in a specified interval. The functional relations may be concisely represented by a three-parameter equation:

$$
\begin{aligned}
h_{n}(t) & =A(n) \cdot M(t) & & \\
A(n) & =a / n & & \\
M(t) & =0, & & \text { if } t<c \\
& =(t-c) /(k-c) & & \text { if } c \leq t \leq k \\
& =1 & & \text { if } t>k .
\end{aligned}
$$

For the data in Fig. $3 \mathrm{~b}, a \approx 28$ bits/s is an asymptotic rate parameter, quantifying the detection rate for targets not yet detected at any time $t$ after onset of the target motion ${ }^{6}$; and $c$ $\approx 0.42 \mathrm{~s}$ and $k \approx 0.86 \mathrm{~s}$ are time constants specifying the start and end of visual motion integration. The function $M(t)$ is simply proportional to elapsed time in the interval $c \leq t \leq k$, with a value of 0 before and 1 after this interval. The primary

\footnotetext{
${ }^{6}$ Estimation and meaning of the detection rate parameter, $a$ : This parameter was estimated by slopes of the integrated hazard functions: $a(t)=[H(t+\Delta t)-H(t)] / \Delta t=-\log _{2}[S(t+\Delta t) / S(t)] / \Delta t$. By hypothesis, the ratio $S(t+\Delta t) / S(t)=s(\Delta t)$ is a constant, invariant with $t$. Thus, $a=$ $\left(-\log _{2} s(\Delta t)\right) / \Delta t$.
}

determinant of detection rates was the capacity parameter $a$, the rate of visual awareness.

Equations 3, 4, and 5 are intended as both a description and a theoretical explanation of the obtained results. These lawful relations involve the hazard rates, not the RT distributions as such. Indeed, the parameters of both visual awareness and motion integration were invariant with mean RT. The lawfulness and generality of these relationships documents the power of hazard rates for measuring the underlying processes.

The lawful relations described by these equations were not anticipated before this experiment. The finding that a single rate capacity parameter described detection rates over wide variations in Set Size, motion information, and RT is a new result. Aspects of the results are consistent with some previous results and theories, of course, but the principal features of the results are new. The General Discussion will consider specific comparisons of these findings with previous results and theories.

\section{Experiment 2}

Experiment 2 tested the generality of these phenomena by evaluating the effects of changes in optical input information on the two hypothesized visual processes.

First, we evaluated effects of variations in motion speed. Three Motion Speeds were 1.40, 2.79, and 4.19\%, as compared to $2.79^{\circ} \mathrm{s}$ in Experiment 1 . Motion Speed affects the spatial length of target motion within a given time, and this spatial information would be expected to affect detection rates. Pilot work confirmed this expectation. How, then, does this variation in visual information influence the two underlying visual processes?

Second, the Set Size of potential targets was expanded to evaluate detection performance for smaller and larger sets of objects: $n=\{1,4,12\}$. For $n=1$, there is no uncertainty about which object will be the target; the only uncertainty is temporal. At the other extreme, patterns of 12 independently moving objects have substantially greater spatial uncertainty and optical complexity, with potential effects of visual crowding. Can a single detection rate parameter apply to Set Sizes 1 and 12 as well as 4? A related question concerns the possible invariance of Motion Integration with such large variations in Set Size.

\section{Method}

Methods and procedures were essentially the same as in Experiment 1, with the following exceptions. First, the observation period for detecting target motions was lengthened from $3 \mathrm{~min}$ to $4 \mathrm{~min}$. Second, the probability of a target motion at any given moment was reduced from Experiment 1 averaging about $13 / \mathrm{min}$ in the present experiment, as compared to about $18 / \mathrm{min}$ in the previous experiment, averaging about 51 target motions in each 4-min observation period. 
Third, Motion Speed varied between sessions - 1.40, 2.79, or $4.20^{\circ} \mathrm{s}$, in counterbalanced order for successive sessions, with order randomized between observers.

Each session consisted of three 4-min observation periods, one for each of the three Set Sizes. With a short break between each of these observation periods, each session took less than $20 \mathrm{~min}$. Observers usually collected data for three such brief sessions in a single sitting. Each observer participated in 18 sessions, six sessions for each of the nine Speed x Set Size conditions. On average, each observer made 304 correct detection responses (range $=247-353$ ) in each of the nine conditions. The number of responses by each observer in each condition was about half that in Experiment 1, resulting in greater variability.

Four observers participated in Experiment 2, all of whom had participated in Experiment 1. (Observer BD in Experiment 1 was no longer available.)

\section{Results and discussion of Experiment 2}

A graphic summary of the results is given in Fig. 5, which shows the hazard rates for the three Set Size conditions for each Motion Speed, based on the combined data for all four observers. Table 3 gives the estimated parameter values of the two-process model for each observer in each Motion Speed condition.

\section{Hazard rates for Set Sizes 1-12 were described by the} same equations As may be seen, variations in hazard rates are well described by the same two-process model as in Experiment 1, extended here to a wider range of Set Sizes and Motion Speeds. The same model described hazard rates in both the Set Size 1 and Set Size 12 conditions - even though the hazard rates of these conditions differed by about 12-fold, occurred in different phases of the detection process, and had median RTs differing on average by more than $1 \mathrm{~s}$. Hazard rates for Set Size 4 involved both phases of the detection process, increasing for $c \leq t \leq k$, and approximately constant with a value of $a / 4$ for $t>k$.

Rate of awareness increased with Motion Speed Figure 5 shows that the rate of visual awareness, $A(n)$, was strongly influenced by Motion Speed. Threefold variations in speed, from 1.40 to $4.19^{\circ} / \mathrm{s}$, caused twofold variations in the rate parameter $a$ for the combined-observer data in Fig. 5. All four observers exhibited similar effects, as seen in Table 3.

Faster speeds produced shorter motion integration Figure 5 suggests that Motion Speed may have had little influence on the duration of motion integration. Indeed, a preliminary data analysis found that a very large percentage of the variance in hazard rates $\left(h_{n}(t)\right)$ could be described with the awareness rate parameter $a$ as the only free parameter, using the parameter values for $c$ and $k$ estimated in Experiment 1 for all three Motion Speed conditions in Experiment 2. This initial analysis was misleading, however. Parameter $k$ has little influence on predicted hazard rates except in a small temporal region near the end of the integration period.

When the effect of Motion Speed on motion integration was evaluated more directly, independently of the rate parameter $a$ (see Appendix 2), we found that speed had a similar and complementary influence on both motion integration and visual awareness. Because Motion Speed had no reliable effect on the onset of motion integration, at time $c$, we independently evaluated the effects of Motion Speed on parameters $k$ and $a$,
Fig. 5 Hazard functions for each of the nine Set Size x Motion Speed conditions, based on the combined RT distributions of all four observers. The two-process model given by the two black lines in each panel is the same as in Figs. $3 b$ and 4, with independently estimated parameters. (See text and Appendix 2 for the estimation procedure)

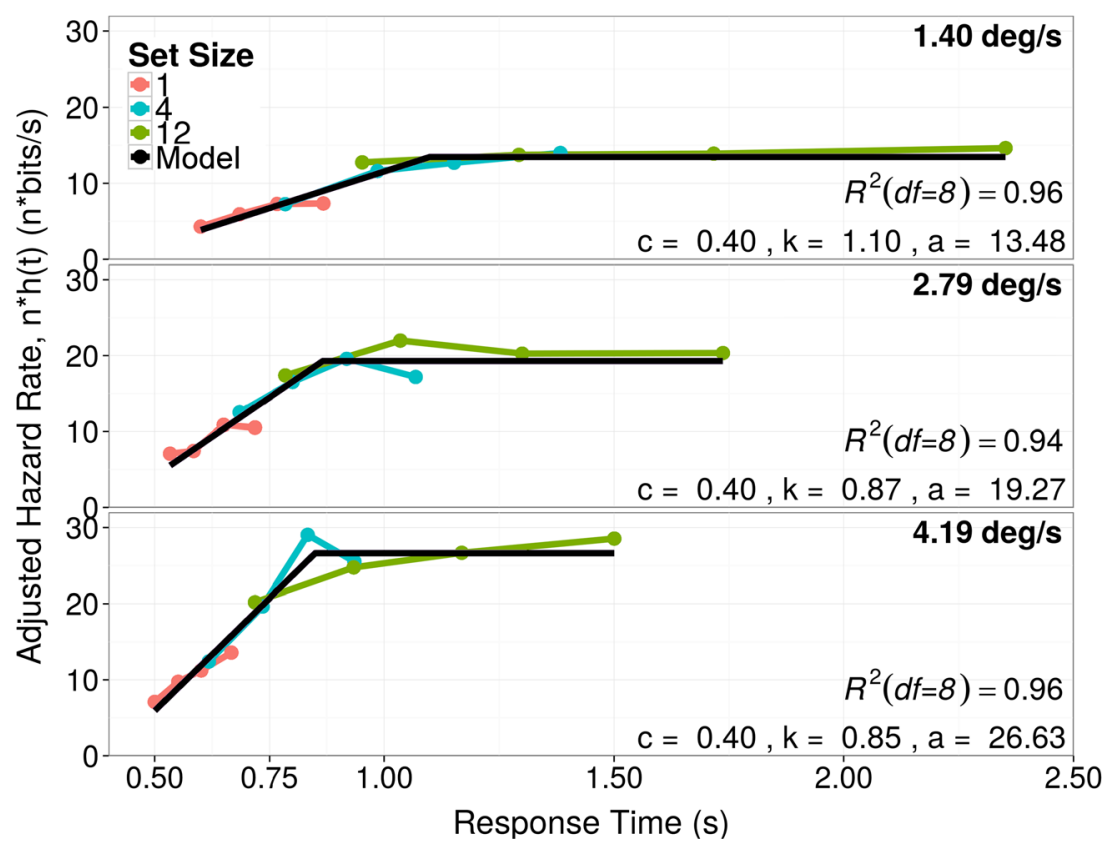


Table 3 Estimated parameters and adjusted $R^{2}(d f=8)$ measures of fit for the hazard rates $\left(h_{n}(t)\right)$ for each observer and Motion Speed condition of Experiment 2 (See text for parameter estimation method)

\begin{tabular}{lllllll}
\hline Observer & Speed $(\% / \mathrm{s})$ & $c(\mathrm{~s})$ & $k(\mathrm{~s})$ & $k-c(\mathrm{~s})$ & $a($ bits/s) & $R^{2}$ \\
\hline AS & 1.40 & 0.45 & 0.84 & 0.39 & 13.0 & 0.98 \\
& 2.79 & 0.45 & 0.69 & 0.24 & 20.0 & 0.95 \\
& 4.19 & 0.45 & 0.64 & 0.19 & 26.7 & 0.94 \\
& Ave. & 0.45 & 0.72 & 0.27 & & 0.96 \\
JL & 1.40 & 0.40 & 0.92 & 0.52 & 15.2 & 0.95 \\
& 2.79 & 0.40 & 0.90 & 0.50 & 20.7 & 0.95 \\
& 4.19 & 0.40 & 0.76 & 0.36 & 25.7 & 0.92 \\
& Ave. & 0.40 & 0.86 & 0.46 & & 0.94 \\
DM & 1.40 & 0.47 & 1.48 & 1.01 & 11.9 & 0.90 \\
& 2.79 & 0.47 & 0.99 & 0.52 & 16.5 & 0.76 \\
& 4.19 & 0.47 & 0.93 & 0.46 & 24.1 & 0.93 \\
& Ave. & 0.47 & 1.13 & 0.66 & & 0.86 \\
KD & 1.40 & 0.40 & 0.97 & 0.57 & 19.5 & 0.99 \\
& 2.79 & 0.40 & 0.80 & 0.40 & 27.7 & 0.96 \\
& 4.19 & 0.40 & 0.72 & 0.32 & 32.0 & 0.92 \\
& Ave. & 0.40 & 0.83 & 0.43 & & 0.96 \\
& 1.40 & 0.43 & 1.05 & 0.62 & 14.9 & 0.96 \\
Ave. & 2.79 & 0.43 & 0.85 & 0.42 & 21.2 & 0.91 \\
& 4.19 & 0.43 & 0.76 & 0.33 & 27.1 & 0.93 \\
& Ave. & 0.43 & 0.89 & 0.46 & & 0.93 \\
\hline \multirow{4}{*}{ A } & & & & &
\end{tabular}

as described in Appendix 2. When Motion Speed increased, the rate of visual awareness increased and the estimated duration of motion integration was found to decrease. Table 3 gives the estimated integration durations $(k-c)$ and awareness rates (a) for each Motion Speed and each observer. For each of the four observers, the longest integration $(k-c)$ occurred when

Motion Speed $=1.40 \%$ and the shortest integration occurred when Motion Speed $=4.19^{\circ} / \mathrm{s}$. The average estimated integration durations for Speeds $1.40,2.79$, and $4.19^{\circ} / \mathrm{s}$ respectively, were 620,420 , and $330 \mathrm{~ms}$. These effects were statistically reliable - by Friedman's two-way analysis of variance by ranks, using the four observers as independent replications of the effects of Speed, $F_{r}=8.00, p \leq 0.01$.

Nevertheless, the rate of visual awareness, $A(n)$, accounts for most of the variance in obtained hazard rates, with little influence of the temporal parameters of motion integration, $M(t)$. Regardless of the effects of Motion Speed, the rate of visual awareness remained inversely proportional to Set Size, $n$, throughout the detection process. The effects of Set Size and Motion Speed were functionally independent and multiplicative.

Spatial and temporal dimensions of motion Faster motions travel greater spatial lengths per unit time, offering a greater rate of spatial information. Faster detection rates for faster speeds are not surprising. Indeed, one can quantify the effects of Motion Speed in spatial as well as temporal units - in bits ${ }^{\circ}$ of visual angle. Dividing the time-based detection rates in bits/s by velocity, in $\%$, yields space-based measures in bits ${ }^{\circ}$. Thus, for the three successively faster Speeds, the time-based scale in Fig. 5 (13.5, 19.3, and 26.6 bits/s) becomes 9.6, 6.9, and 6.4 bits $/^{\circ}$. Reduced variations in the space-based measures as compared to the time-based measures indicate that detection performance was influenced more strongly by the spatial extent than the temporal extent of motion. Nevertheless, time also matters. Increased Speeds produced lower space-based detection rates because the time per unit space was reduced.

A difference in the results of Experiments 1 and 2 Detection rates for Motion Speed $2.79 \%$ s were lower in Experiment 2 than Experiment 1. The awareness rate parameter for these four observers averaged $a=21.3 \mathrm{bits} / \mathrm{s}$ in Experiment 2, but was $a=27.4 \mathrm{bits} / \mathrm{s}$ in Experiment 1. All four observers exhibited a lower rate of visual awareness in Experiment 2 than Experiment 1, although the difference was small for observer $\mathrm{KD}-28.5$ and 27.4 bits $/ \mathrm{s}$ in Experiments 1 and 2 respectively. The reason for this difference is not known. One possible cause could be that the relative rates of target events were lower in Experiment 2 - about 13/min, as compared to 18/min in Experiment 1. Subjectively, this difference in target rates seemed inconsequential, and its effect was not noticed before data analysis. The potential effect of the target rate variable is one of several questions requiring additional investigation.

\section{Experiment 3}

Experiment 3 addressed a question about the target motion information in this study. Was the moving "prey" object a basic element of the target motion, or was the target defined by its approximately linear trajectory? Does the moving "prey" object enhance target detection, or might it even compete and interfere with recognition of the target motion?

Experiment 3 tested this question by comparing detection performance with and without a visible prey object. In an Invisible Prey condition, the red prey object was simply not displayed, while the target motion was directed toward the same slowly moving positions as in the Visible Prey condition. The Visible Prey condition was the same as in Experiments 1 and 2. Random background motions were the same in both conditions.

\section{Method}

As in Experiments 1 and 2, Set Size determined the task difficulty. Four Set Sizes in Experiment 3 were $n=\{1,2,4,8\}$.

Results were obtained for three observers who had also served in Experiments 1 and 2 - JL, DM, and KD. Each observer served in eight sessions. Each session was devoted to either the Visible Prey or the Invisible Prey condition, 
which occurred in counterbalanced order. As in Experiments 1 and 2, each session contained four blocks of trials, one block for each Set Size, which occurred in a randomly permuted order in each session. An average of about 210 target events occurred for each observer in each of the eight conditions. The amount of data for each observer and condition was reduced in this experiment but sufficient for comparing performance in the Visible and Invisible Prey conditions. Estimated hazard rates were less reliable, however, than in Experiments 1 and 2.

\section{Results and discussion of Experiment 3}

A graphic summary of the results is given in Figs. 6 and 7. Table 4 lists the mean RTs, hit rates, and false alarms for each observer in each of the eight conditions.

Hazard rates were consistently lower in the Invisible Prey condition (Fig. 6). Hit rates were also lower and false alarm probabilities consistently higher in the Invisible Prey condition (Table 4). Thus, visibility of the "prey" location aided target detection - by adding information about the direction of the target motion. Adding information about the prey might conceivably have competed for visual attention and hindered detection, but this did not happen. The result is interesting but not surprising.

Figure 6 shows "adjusted" hazard rates, $n \bullet h_{n}(t)$, as a function of Set Size, with separate graphs for the Visible and Invisible Prey conditions. The solid black lines describe the two-process model developed in Experiment 1, fit to the hazard rates for the Visible Prey condition combined over the three observers. The model estimation procedure was the same as in Experiment 1, minimizing standardized squared differences between observed and predicted values of the hazard rates, $\left[h_{n}(t)-P_{n}(t)\right]^{2} / P_{n}(t)$, at each of the 16 data points. The estimated parameter values were $a=24.2 \mathrm{bits} / \mathrm{s}, c=0.40 \mathrm{~s}$, and $k=0.93 \mathrm{~s}$. This model accounted for $91 \%$ of the variance

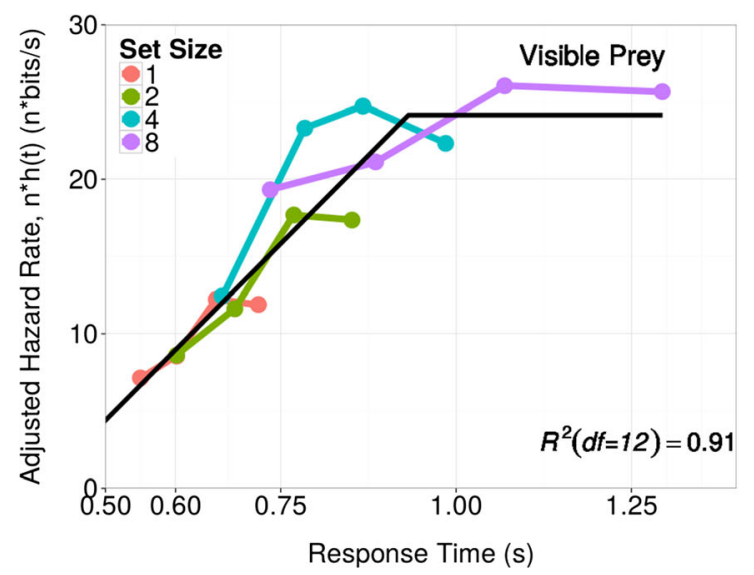

Fig. 6 Effects of Set Size on the adjusted hazard functions, $n \cdot h_{n}(t)$, for the Visible Prey and Invisible Prey conditions of Experiment 3. Plotted data are from the combined RT distributions of three observers. The solid black lines describe the predicted hazard rates for the Visible Prey
$\left(R^{2}=0.908, d f=12\right)$ in hazard rates for the Visible Prey condition. The same descriptive model is shown for both Visible and Invisible Prey conditions, to facilitate visual comparison between the two conditions.

Not surprisingly, due to the reduced amount of data for individuals (average of 203 correct responses for each Set Size in the Visible Prey condition), the hazard functions for individual observers in the Visible Prey condition were more variable than in Experiments 1 and 2. (For observers JL, DM, and $\mathrm{KD}$, respectively, the model accounted for $68 \%, 86 \%$, and $81 \%$ of the variance; but the estimated awareness capacity parameters were consistent with those in Experiment 2: $a=$ $\{26.0,23.0,26.0\}$.) Each individual observer performed consistently worse in the Invisible Prey conditions.

Data for the Invisible Prey condition were not well described by this three-parameter model. When these three parameters were estimated for response times in the Invisible Prey condition, they accounted for only $54 \%$ of the variance of the obtained hazard rates. Apparently, ambiguities of motions toward an invisible prey disrupted the consistency of visual processes observed in the Visible Prey conditions. RTs were longer and probabilities of missed detections and false alarms were also much higher in the Invisible Prey condition. Because RTs are not defined for misses and false alarms, the influence of error-related factors cannot be identified in the hazard rates for the Invisible Prey condition.

The hazard rates in Fig. 6 do not offer a clear quantitative comparison of performance in the Visible and Invisible Prey condition - partly because the hazard rates were evaluated at different times for the two conditions. A clearer evaluation of the performance difference between these two conditions was obtained from half-life times measured at the same RTs for both Visible and Invisible Prey conditions. For each of the four Set Size conditions, the survivor function was evaluated for a single RT distribution combined from both Visible and

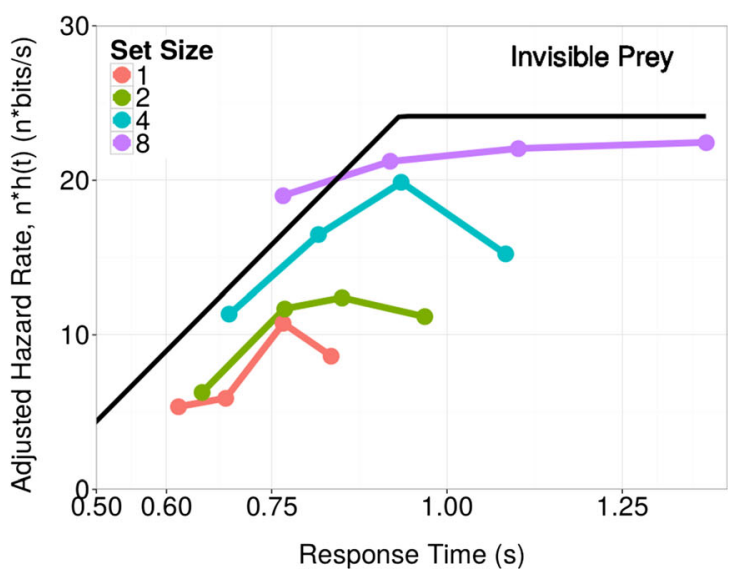

condition, with the same model shown for both left and right graphs respectively. Estimated parameter values were $a=24.2 \mathrm{bits} / \mathrm{s}, c=$ $0.40 \mathrm{~s}$, and $k=0.93 \mathrm{~s}$ 
Invisible Prey conditions, using the four time values at which $S(t)_{\text {Combined }}=\{0.90,0.70,0.50,0.30\}$. At all of these times, the percentage of survivors was lower for the Visible than for the Invisible Prey condition. Using these four time values, we then computed the half-life times for the Visible and Invisible Prey conditions separately. The half-life times are the added response times at which the number of survivors was reduced by one half.

Figure 7 shows these half-life detection times for the Visible and Invisible Prey conditions in each Set Size condition. Underlying data are the same as in Fig. 6. These timebased measures, however, are (a) reciprocals of the hazard rates, $1000 / h_{n}(t)$, in units of $\mathrm{ms} /$ bit, (b) estimated by the empirical half-life of targets undetected at a given time $t$, and (c) estimated at the same RT values for both Visible and Invisible Prey conditions. Figure 7 complements the description of detection rates shown in Fig. 6, using units of time, ms/bit.

As may be seen, half-life detection times were consistently faster for the Visible Prey than for the Invisible Prey condition. Interestingly, time differences in all four Set Size condition averaged about $50 \mathrm{~ms}$ longer in the Invisible Prey conditions, despite large variations in RT across the four Set Size conditions — averaging 47, 54, 56, and $50 \mathrm{~ms} /$ bit differences for Set Sizes $1,2,4$, and 8 , respectively. Each observer provided 16 comparisons of half-life detection times for the Visible and Invisible Prey conditions (4 RTs X 4 Set Sizes). Across observers, halflife times were longer in the Invisible Prey condition in 43 of the 48 comparisons $(15 / 16,12 / 16$, and 16/16 for observers JL, $\mathrm{DM}$, and $\mathrm{KD}$, respectively). These results plus the accuracy data in Table 4 demonstrate that detection performance was consistently less efficient for the Invisible Prey condition.

\section{General discussion}

This study found that a constant rate of visual awareness was consistent with widely varying rates of target detection obtained over wide ranges of Set Sizes, performance levels, response times, and motion information. The effects of divided attention on the temporal process of target detection were very large, but surprisingly simple and consistent.

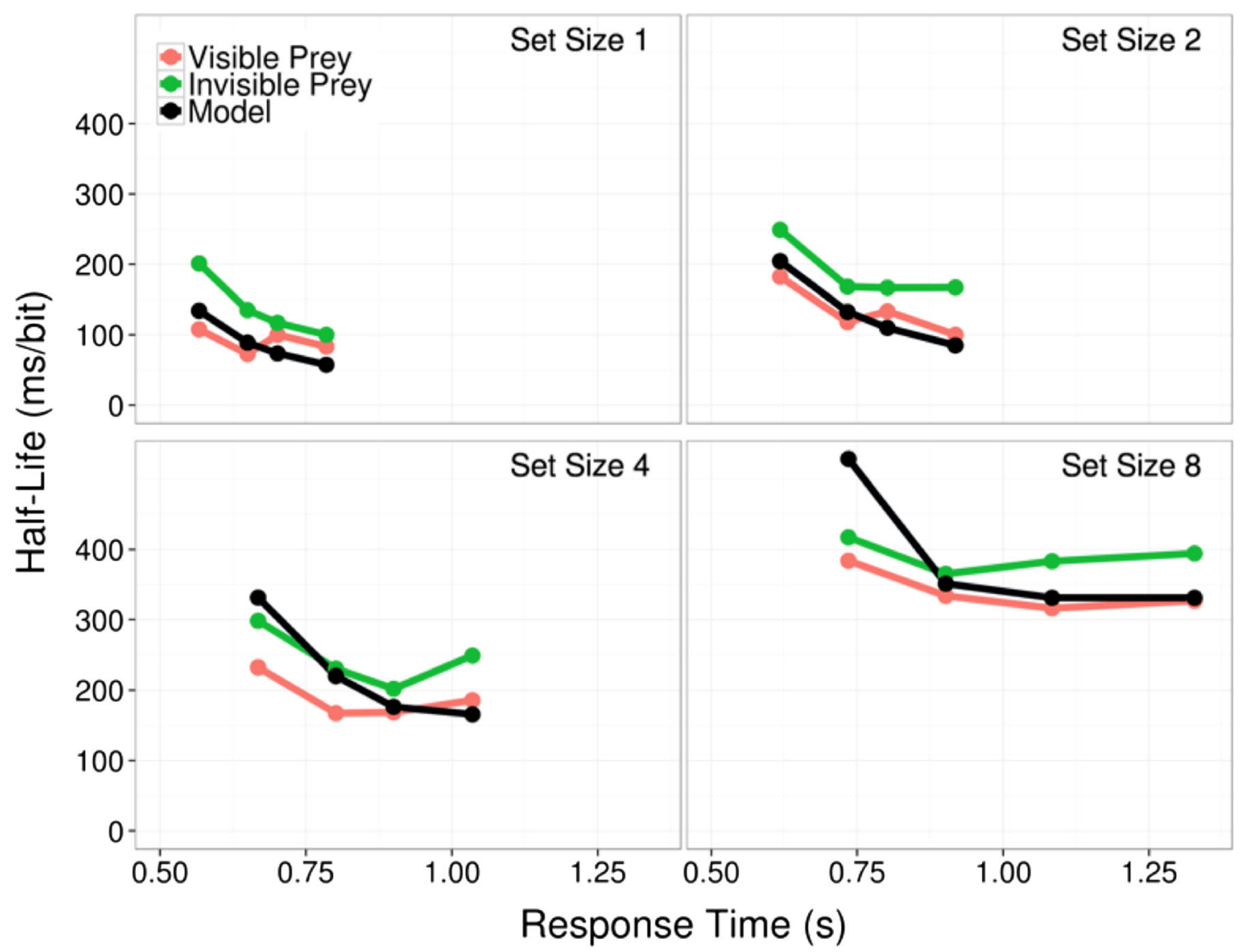

Fig. 7 Detection half-life times (ms/bit) for the Visible and Invisible Prey conditions at each Set Size condition in Experiment 3. Predicted values are from the same model and parameter values as in Fig. 6. The data patterns differ between Figs. 6 and 7 because (a) the scales of hazard rates in Fig. 6 and half-life times in Fig. 7 are reciprocal; (b) at the first two RTs, half-life times were computed over longer time intervals than for the hazard rates; and (c) half-life times in Fig. 7 were computed at different RT values from those in Fig. 6. Discrepancies between the obtained and predicted times for Set Sizes 4 and 8 result from estimating half-life times over large ranges of RT that involved responses from both increasing and asymptotic hazard rates 
Table 4 Hit Rates (HR), proportion of False Alarms among total detection responses (FA/(Hit+FA)), and Mean RTs (ms) for each observer and condition in Experiment 3

\begin{tabular}{|c|c|c|c|c|c|}
\hline Set Size & Prey & Observer & Hit Rate & $\mathrm{p}(\mathrm{FA} \mid \mathrm{r})$ & Mean RT \\
\hline \multirow[t]{8}{*}{1} & \multirow[t]{4}{*}{ Visible } & JL & 1.00 & 0.04 & 638 \\
\hline & & DM & 1.00 & 0.02 & 747 \\
\hline & & $\mathrm{KD}$ & 1.00 & 0.01 & 665 \\
\hline & & Average & 1.000 & 0.026 & 683 \\
\hline & \multirow[t]{4}{*}{ Invisible } & $\mathrm{JL}$ & 0.99 & 0.12 & 734 \\
\hline & & $\mathrm{DM}$ & 1.00 & 0.03 & 859 \\
\hline & & $\mathrm{KD}$ & 0.99 & 0.00 & 766 \\
\hline & & Average & 0.996 & 0.052 & 786 \\
\hline \multirow[t]{8}{*}{2} & \multirow[t]{4}{*}{ Visible } & JL & 1.00 & 0.10 & 784 \\
\hline & & DM & 1.00 & 0.03 & 844 \\
\hline & & $\mathrm{KD}$ & 1.00 & 0.03 & 769 \\
\hline & & Average & 1.000 & 0.051 & 799 \\
\hline & \multirow[t]{4}{*}{ Invisible } & JL & 0.98 & 0.09 & 939 \\
\hline & & $\mathrm{DM}$ & 0.98 & 0.07 & 883 \\
\hline & & $\mathrm{KD}$ & 0.98 & 0.01 & 917 \\
\hline & & Average & 0.982 & 0.058 & 913 \\
\hline \multirow[t]{8}{*}{4} & \multirow[t]{4}{*}{ Visible } & JL & 0.99 & 0.04 & 941 \\
\hline & & $\mathrm{DM}$ & 0.99 & 0.03 & 960 \\
\hline & & $\mathrm{KD}$ & 1.00 & 0.01 & 865 \\
\hline & & Average & 0.992 & 0.028 & 922 \\
\hline & \multirow[t]{4}{*}{ Invisible } & JL & 0.91 & 0.09 & 1,045 \\
\hline & & $\mathrm{DM}$ & 0.93 & 0.08 & 1,025 \\
\hline & & $\mathrm{KD}$ & 0.95 & 0.04 & 1,026 \\
\hline & & Average & 0.930 & 0.070 & 1,032 \\
\hline \multirow[t]{8}{*}{8} & \multirow[t]{4}{*}{ Visible } & JL & 1.00 & 0.02 & 1,209 \\
\hline & & $\mathrm{DM}$ & 0.99 & 0.06 & 1,228 \\
\hline & & $\mathrm{KD}$ & 1.00 & 0.01 & 1,122 \\
\hline & & Average & 0.994 & 0.029 & 1,186 \\
\hline & \multirow[t]{4}{*}{ Invisible } & JL & 0.82 & 0.07 & 1,286 \\
\hline & & $\mathrm{DM}$ & 0.81 & 0.15 & 1,162 \\
\hline & & $\mathrm{KD}$ & 0.85 & 0.04 & 1,262 \\
\hline & & Average & 0.826 & 0.083 & 1,237 \\
\hline
\end{tabular}

The lawful structure of these phenomena was revealed by hazard rates, involving temporal derivatives of the detection RTs. Rates of change in detection probability were more informative than detection probabilities as a function of time. A significant advantage of the hazard rate measures, as compared to the underlying RT probability distributions, is that they provide a ratio scale of relative performance in different conditions and at different times. Such ratios were essential to the present results.

Importantly, the hazard rates in this study revealed effects of Set Size that were not only different but also simpler, more precise, and more lawful than those described by the relation between Set Size and mean RT. Comparison of Figs. 2 and 3 illustrates how an experimenter's choice of performance measure can influence the resulting picture of phenomena and processes. How would evaluations of hazard rates alter interpretations of previous visual search experiments based on mean RTs? The answer is not known.

The novel results of this study as compared to those of other related experiments on visual attention, search, motion perception, and performance may also reflect methodological differences. In particular, experiments with discrete trials and predictable distinct target onsets add non-visual information about the occurrence of a target, and such information would likely affect response criteria and RT distributions in some studies of visual search. Target onset information in the present study, however, was entirely visual and driven by temporally increasing differences between target and background motions.

Equations 3-5 and Figs. 3b, 4, and 5 constitute a descriptive model of the results. To clarify the rationale for this descriptive model, the empirical observations on which it is based are listed below. These empirical observations involve interpolations and extrapolations of the hazard rates evaluated at local RTs. The wide range of obtained hazard rates were closely approximated (within small random error) by a simple continuous function of time given by the product of two functions, one a function of Set Size $(n)$ and the other a function of RT $(t)$. Three parameters of this descriptive model were estimated without additional assumptions beyond the approximation, interpolation, and extrapolation of empirical hazard rates.

Empirical observations The following statements indicate the empirical basis for the abstracted general description of the results.

(1) If $h_{i}(t)$ and $h_{j}(t)$ are the momentary hazard rates (interpolated or extrapolated) for two conditions with Set Sizes $i$, $j=\{1,2,4,6,8,12\}$, then the ratio of these two hazard rates approximately equaled the reciprocal of those two Set Sizes: $h_{i}(t) / h_{j}(t) \approx j / i$ [with different Set Sizes and hazard rates, $h_{n}(t)$ in different experiments].

(2) The equivalence of relative hazard rates and relative Set Sizes, described in observation (1), was invariant with RT $(t)$ and with changes in hazard rates corresponding to changes in the duration and length of motion.

(3a) For RTs within the temporal interval $c \leq t \leq k$, hazard rates were approximately proportional to the temporal duration, $(t-c) /(k-c)$, where $c$ and $k$ are RTs (in units of s). That is, if $t$ and $u$ are two RTs in the interval $(c, k)$, then $h_{n}(t) / h_{n}(u) \approx(t-c) /(u-c)$.

(3b) If RT $>k$, then hazard rates were constant and proportional to Set Size, $n-$ i.e., $h_{n}(t)=a / n$, where the constant parameter $a$ is quantified in bits/s. 
(3c) The time parameters, $c$ and $k$, were invariant with Set Size.

Corollaries and inferences based on these empirical statements include:

(C1) Two functionally independent and temporally concurrent processes were identified by the selective influence of different experimental variables. One process, termed Visual Awareness, was a function of Set Size $n$, but was invariant with time; and a second process, termed Motion Integration, was a function of time $t$, but invariant with Set Size.

(C1a) A detection rate capacity parameter, $a$, was invariant with Set Size $(n)$ and RT $(t)$. The detection process governed by Set Size $(n)$ and the rate capacity parameter $a$ was represented symbolically as

$A(n)=a / n$.

The concept of "Visual Awareness" was based partly on the finding that for RTs $>k$, hazard rates remained constant - despite longer durations of target motion, maintaining hazard rates at the level given by target information visually integrated up to time $k$. The "awareness" concept also corresponded to the subjective impression that sometimes, on trials with slow RTs, when we finally detected the target motion we realized that it had been moving toward the "prey" for an extended time and distance. Thus, we must have "seen" the target motion, but, paradoxically, failed to consciously notice and detect it.

(C1b) A time-dependent visual process was characterized by temporal parameters $c$ and $k$, which are values of RT. These parameter values were invariant with Set Size and mean RT. The process is described by the simple equations:

$$
\begin{array}{ll}
M(t)=(t-c) /(k-c), & \text { if } c \leq t \leq k ; \\
M(t)=0, & \text { if } t<c ; \\
M(t)=1, & \text { if } t>k .
\end{array}
$$

The proportionality of hazard rates and elapsed time between $c$ and $k$ corresponds to the increase in optical target information with increasing duration and length of motion.

(C1c) A wide range of hazard rates found in Experiments 1, 2, and in the "Visible Prey" condition of Experiment 3, covering a range of Set Sizes, RTs, and Motion Speeds were well characterized by a product of two simple linear functions,

$$
h_{n}(t)=A(n) \cdot M(t) \text {, }
$$

where the two functions $A(n)$ and $M(t)$ are defined by Equations (4) and (5) above. (Parameter $a$ varied substantially with Motion Speed in Experiment 2, and differed slightly but significantly for the same Motion Speed in Experiments 1 versus 2 and 3. Parameter $k$ also varied with Motion Speed, but Motion Speed did not affect the temporal parameter c.)

(C1d) The same parameters cannot account for the effects of both Set Size $(n)$ and time $(t)$. The joint effects of these two functionally independent factors must involve two independent visual processes rather than one process.

(C2) The temporal process of target detection rates, characterized by parameters $a, c$, and $k$, was invariant over large changes in the RT means and variances.

The same equations and same three parameter values, $a, c$, and $k$, described the hazard functions for entirely different RT distributions produced by different Set Sizes. This finding was especially striking in Experiment 2, where the RT distributions for Set Sizes 1 and 12 had only negligible overlap.

(C3) The temporal structure of the target detection process was defined on hazard functions - on the differential structure of log survivor functions - but not on the RT distributions as such.

Equation (3) describing the obtained hazard rates as a function of Set Size and RT does not describe the RT probability distributions. Hazard rates are conditioned on the survival function at a given time $t$, but they do not specify the survival probability at that time.

The hazard rate measure The RT distributions in these experiments were highly variable, offering an uncertain index of target detection speeds. We explored the processes underlying these distributions by applying theoretical research by Townsend and colleagues on hazard functions and perceptual capacity (e.g., Houpt \& Townsend, 2012; Townsend \& Ashby, 1983; Townsend \& Eidels, 2011; Townsend \& Nozawa, 1995; Townsend \& Wenger, 2004b; Wenger \& Gibson, 2004). The hazard functions revealed a clear picture that was not evident in the RT means and variances. Indeed, a basic result implicit in these data is that the structure of the hazard functions was invariant with substantial changes in the RT distributions. That invariance was an important secondary finding of this study.

Hazard rates $h(t)$ measure the detection rate at any given time $t$ after target onset. The bits/s units offer convenient terminology and interpretation, but the base of the logarithms is otherwise arbitrary. The reciprocal measure in temporal units of s/bit is the "half-life" of targets not yet detected at time $t$ 
i.e., the additional time needed to reduce the number of undetected targets by half. The half-life measure is directly proportional to the time constant $\tau$ commonly used to quantify exponential decay functions.

An important advantage of hazard functions is that they provide scale-free (dimensionless) ratios of relative performance at different times and in different conditions. Such ratios were essential to the present findings.

Hazard rates in this study were estimated from the slopes of integrated hazard functions, $H(t)$. And these slopes were evaluated at four intervals defined by $20 \%$ increments in the CDF, from the $10^{\text {th }}$ to $90^{\text {th }}$ percentiles. That is, $h(t)=\Delta H(t) / \Delta t$, as described above. (Fig. 9 in Appendix 1 shows hazard functions defined in much the same way, but evaluated at $5 \%$ intervals from 5 th to 95 th percentiles, using a running window of $20 \%$ differences in the RT CDFs.) Details of this analysis depart from the methods developed by Townsend and colleagues, though these departures involve secondary details but not fundamental rationale. The specific methods adopted here evolved by exploring and comparing several different analyses of the obtained data. No doubt, different methods may be needed to reveal different relational structures of data from different experimental methods.

Two departures of the present measurement methods from previous methods may be noted. First, descriptive analyses of the momentary hazard rates, $h(t)$, were found to represent our results more clearly than the integrated hazard functions, $H(t)$, investigated by Townsend and colleagues (e.g., Houpt \& Townsend, 2012; Townsend \& Eidels, 2011; Townsend \& Wenger, 2004a, b; Wenger \& Gibson, 2004). A principal advantage of the momentary hazard rates for our study is that they revealed a temporal process quantified by the detection rates at one time relative to another. Ratios of integrated hazard functions at given times would not have revealed this temporal structure of the detection process. Moreover, temporal variations in ratios of integrated hazard functions in Experiment 1 gave a misleading description of the relative detection rates for Set Size 2 as compared with Set Sizes 4, 6, and 8 - as demonstrated and examined in Appendix 1.

Second, hazard rates were evaluated at times identified by specific quantiles of the RT distribution function (CDF) rather than evaluating and comparing hazard functions at the same times for different conditions. Exploratory analyses found that comparisons between conditions were sometimes unreliable when the same time bins were used for multiple conditions, due to large differences in response frequencies for different conditions in the same time intervals. These differences in RT distributions for different Set Size conditions were especially striking in Experiment 2, where RT distributions for Set Sizes 1 and 12 had negligible overlap. Despite these differences in the RT distributions for different Set Size conditions, a common temporal structure emerged from comparisons between hazard rates based on quantiles of the RT distributions. The specific number and intervals of quantiles at which to evaluate the hazard functions were ad hoc, based on exploratory comparisons, but this procedure may not be best in general.

The rate of visual awareness The rate of visual awareness was the primary determinant of detection performance in this task. The process of awareness was quantified by the function, $A(n)=a / n$, where $a$ is a constant rate (in bits/s) and $n$ is the number of observed objects. The rate parameter $a$ quantifies the capacity limit of a time-shared system in which the time to detect any individual item increases in direct proportion to the number of items being processed (see Townsend \& Ashby, 1983, pp. 85-88; Townsend \& Nozawa, 1995, p. 334). To our knowledge, a similar capacity limit has not been found in previous studies of response time. Hyman's (1953) classic study of the dependence of mean RT on information transmitted is analogous, but the task variables and quantitative results were quite different. Here, Set Size had large, linear effects on mean RT, but the relative hazard rate at any given time was approximated by $a / n$. In Experiment 1, this awareness rate parameter was $a \approx 28 \mathrm{bits} / \mathrm{s}$. This rate parameter was constant over time; it did not determine the RT distribution as such. The pervasive influence of this rate capacity suggests that it might reflect a conserved property of neurophysiological matter and energy, but evidence about such physical grounding is not yet available.

Experiment 2 found that this parameter depended on Motion Speed, ranging about $12-30 \mathrm{bits} / \mathrm{s}$ for motions of $1.4-4.2 \%$. This effect of Motion Speed implies that the limited rate of visual awareness and detection was spatiotemporal. One can equally well express the rate of awareness in spatial units, in bits $/^{\circ}$. Expressing the rates in spatial units yields less variation with Motion Speed - indicating that the spatial dimension was more important than the temporal dimension, but both dimensions had influence. The present study offers limited initial evidence about this spatiotemporal dimension of visual awareness, but the issue warrants additional investigation.

Motion speed was not the only influence on the rate of awareness. Experiments 2 and 3 found different values for this capacity parameter than did Experiment 1 for the same Motion Speed. One hypothesis is that the quantitative difference might have been caused by a difference in the target probabilities in these experiments - 18 targets/min in Experiment 1 and $\sim 13 / \mathrm{min}$ in Experiments 2 and 3. Subjectively, the difference in target probability was barely noticeable, and seemed inconsequential. Further investigation is needed.

Importantly, the awareness rate, $a$, was essentially timeinvariant. That is, the detection rate for a not-yet-detected target was independent of how long it had been undetected. A constant hazard rate identifies a stochastic process with exponentially distributed event times. Such constant-rate 
processes are found in many physical phenomena, such as radioactive decay, Brownian motion, and molecular diffusion. Diffusion processes have been shown to account for speed and accuracy in a variety of discrimination, decision-making, and attention tasks (e.g., Ratcliff, 2014; Ratcliff et al., 2016; Smith, 2010; Smith \& Ratcliff, 2009). A diffusion process is also compatible with aspects of the present results.

Unlike previous studies of RT distributions, however, the process of visual awareness was characterized here by the detection rates, not by the RT distributions as such. The hazard rate at a given time is independent of the RT distribution up to that time. Constant hazard rates were found by partitioning the influence of time and Set Size on two parallel processes - motion integration and visual awareness. Constant hazard rates characterized visual awareness but not motion integration. The observed RT distributions have an exponential component that varies with the multiplicative influence of motion integration.

\section{Awareness as a parallel process with a divisible rate Visual} attention to varied Set Sizes had a decisive influence on the rate of target recognition. This influence, however, did not occur at a particular stage in a sequence of processes, contrary to some conceptions of attention. Instead, the influence of attention was uniform throughout the temporal process, operating in parallel with visual integration of motion.

The conclusion that a process of visual awareness operates in parallel with other visual processes is consistent with growing evidence: Observers often fail to notice highly visible optically salient objects or events when they are attending to other aspects of a scenario (e.g., Chabris \& Simons, 2009; Drew et al., 2013; Mack, 2003; Mack \& Rock, 1998; Simons, 2000; Simons \& Chabris, 1999; Wayand et al., 2005). The same limitations of awareness undoubtedly underlie interactions with information technology (Levin \& Baker, 2015; Varakin et al., 2004), effects of distracted driving (McCarley et al., 2004; Strayer et al., 2003), and other forms of distraction (Eriksen \& Johnson, 1964; Stothart et al., 2015).

The present study documents a related phenomenon and illuminates additional characteristics of the process of awareness: First, visual awareness in the present task involved a processing rate that varied with the attentional demands of the context.

Second, the rate of awareness was limited in this task by a constant capacity. A single capacity parameter was invariant across a wide range of Set Sizes, response times, and motion lengths. Evidently, visual awareness involves a capacitylimited parallel process (Townsend \& Ashby, 1983, pp. 8588; Townsend \& Nozawa, 1995, p. 334). This rate capacity was influenced by other factors, such as Motion Speed, though currently available evidence about these factors is limited.
Third, the rate of awareness was evenly divisible by the number of observed objects from which the target was sampled. Thus, the rate of awareness for any given stimulus item depends on the attentional demands of the task environment. The present methods do not reveal variations in levels of awareness nor how such variations might be distributed over space, time, or multiple objects.

Motion integration Hazard functions did increase over time - but the increases occurred within an initial time interval. The effects of time and Set Size were functionally separable - with different parameters for different processes. Motion integration, $M(t)$, varied with time but not Set Size. Motion integration in this study was quite simple - with output information proportional to the elapsed time between $c \approx 0.4 \mathrm{~s}$ and $k \approx 0.9 \mathrm{~s}$. Thus, $M(t)=(t-c) /(k-c)$ for $c \leq t \leq k$, and $M(t)$ $=0$ if $t<c$, and $M(t)=1$ if $t>k$. Within this temporal integration period, hazard rates, $h_{n}(t)$, were directly proportional to the relative elapsed time and inversely proportional to Set Size.

That hazard rates increased over time is certainly not surprising: Spatiotemporal information for discriminating target motions from random background motions was proportional to the time and distance of the target motion. Motion perception entails visual integration over time and space. The function $M(t)$ quantifies this visual integration.

Two other noteworthy findings involved the duration (and spatial length) of motion integration: First, the duration of integration was limited, with RTs $>k$ unaffected by the duration of motion. A possible mechanism is a leaky integrator similar to that proposed for certain decision-making phenomena by Usher and McClelland (2004). If evidence accumulates at a constant rate and also decays at a rate proportional to the cumulative total, then the rates of gain and loss are equal after a certain period.

Second, the integration duration was unaffected by Set Size, despite the large effect of Set Size on detection rates. Thus, if the duration of motion integration reflects a balance of information integration and decay rates, then those integration and decay rates were independent of the Set Size parameter that governed the observed detection rates. Motion integration duration was surprisingly consistent across both Set Size and observers. Evidently, visual awareness and motion integration are functionally independent.

Motion integration duration did vary with Motion Speed, however. Nevertheless, the effect of Motion Speed on the integration duration had only a small influence on detection performance, which depended primarily on the rate of visual awareness, $A(n)$.

How does the obtained linear integration duration of roughly $500 \mathrm{~ms}$ compare with other results in the literature? A similar integration time, about $400-500$ ms was obtained by Watamaniuk et al. (1995) using stimulus patterns and a 
detection task very different from the present study. Linear motions of a single target dot, at speeds of $10-15^{\circ} / \mathrm{s}$, were embedded in dense random motion backgrounds that precluded focused attention to individual dots. Unlike the present study, the moving targets "popped out" visually from the random backgrounds. Watamaniuk et al. evaluated detection accuracies as a function of temporal duration, whereas integration times in the present study were estimated from RTs in a task challenged by the spread of attention. The similar integration durations found in these studies may be coincidental.

The temporal characteristics of motion integration certainly depend on the specific signal/noise properties of a particular target in a particular task. Watson and Turano (1995), for example, found highly non-linear temporal integration characteristics over durations like those in the present study, when detection thresholds for a single Gabor patch were measured in terms of minimum contrast energy. Contrast energy thresholds were minimized for motion durations of $133 \mathrm{~ms}$.

The stimulus and task conditions that yield linear motion integration similar to that in the present study are not yet known.

Multiplicative relationships Multiplicative (and divisive) influence relations were found in two aspects of these data: (1) Attending to multiple objects evenly divided the detection rate for a given target object. That is, $h_{n}(t)=(1 / n) \bullet h_{1}(t)$. Equivalently, detection half-life times $(\mathrm{s} / \mathrm{bit})$, say $l_{n}(t)=1 /$ $h_{n}(t)$, were directly proportional to $n: l_{n}(t)=n \bullet l_{1}(t)$. These relationships held across variations in $t$.

(2) As expressed by the equation $h_{n}(t)=A(n) \cdot M(t)$, the behavioral detection rate at any time $t$ was jointly determined as the numerical product of two concurrent visual functions, labeled visual awareness and motion integration.

The relationships described by Equations 3-5 involve scale-invariant ratios of performance at various times and in various task conditions. The units of time (s), Set Size (n), hazard rates (bits/s), and logarithm base (2) are arbitrary. The simplicity of these lawful relations validates the hazard rate as a fundamental performance measure. The equation $h_{n}(t)=A(n) \cdot M(t)$ is also independent of mean RT.

The multiplicative relations in Equations 3-5 contrast with the additive factors framework often used to model the combined effects of multiple factors on RTs. The additive factors framework is exemplified in statistics by the general linear model for ANOVA and multiple regression. Additive factors models generally assume interval-scale variables, for which products and ratios are not meaningful. The linear relation between Set Size and RT in this study also exemplifies such additive effects. But this linear relation barely hinted at the multiplicative and divisive relations revealed by the hazard rates.

Limited-span models of attention The role of attention in perception and performance often involves limitations of visual working memory, VWM. The limited capacity of VWM has been an important focus of research on attention (e.g., Averbach \& Coriell, 1961; Eriksen \& Lappin, 1967; Fougnie et al., 2010; Luck \& Vogel, 1997; Miller, 1956; Sperling, 1960; Woodman et al., 2007). Visual attention and VWM both have limited capacity, and these capacities are often linked, either implicitly or explicitly.

The limited capacity of visual awareness documented in the present study did not involve limitations of VWM. As in visual search tasks, target detection here was based on optical information, requiring no memory. Spatiotemporal integration was necessary for detecting these target motions, but this motion integration obviously differed from the VWM required by tasks in studies of the span of apprehension.

Motion patterns in this study were adapted from studies of multi-object tracking (e.g., Alvarez \& Franconeri, 2007; Fehd \& Seiffert, 2010; Franconeri et al., 2010; Pylyshyn \& Storm, 1988). Unlike the MOT task, the targets here were optically defined, requiring no memory to specify a target. Performance in MOT tasks exhibits a non-linear dependence on size of the memory-based target set, with a sharp decline for $n>\sim 3$, but the present results exhibited no such non-linearity. In contrast to hypotheses about the limited spans of apprehension and memory, the present results reflected quite different limitations, exhibiting no non-linear effect of the number of attended objects.

Normative statistical theories with unlimited capacity Statistics of the input information are sufficient to explain many reductions in speed and accuracy caused by spreading attention to more objects. Increased Set Size reduces target detection by even unlimited-capacity perceptual systems. Signal detection theory (e.g., Green \& Swets, 1966; Tanner, 1956) and choice theory (e.g., Luce, 1963) offer conceptually related, parsimonious, quantitative theories that predict reduced target detection caused by increased background noise or distractors. Do such normative statistical theories account for the present observations?

The short answer is "No." Quantitative and qualitative aspects of the present Set Size effects differ from the predictable performance effects of changes in input noise. In choice theory, increasing the Set Size increases the probability that a distractor will be mistaken for the target. The predicted effect is a nonlinear function of Set Size: If $D_{1}$ is the choice theory measure of the discrimination between a target and a single distractor $\left(D=-\ln \eta\right.$, a ratio scale measure similar to $\left.d^{\prime}\right)$, and if $D_{n}$ measures discrimination between the target and $n$ equally confusable distractors, then $D_{n}=\ln \left[\left(e^{D 1}+n-1\right) / n\right]$ (see Lappin \& Staller, 1981). A similar nonlinear formula approximates the signal detection theory prediction (Peterson et al., 1954). The proportionality of detection rates and Set Size in the present study, $h_{n}(t)=h_{l}(t) / n$, is inconsistent with these nonlinear predictions. 
Normative theories with noise-limited target detection processes, however, are consistent with the motion integration phase of the present detection process, when hazard rates increased proportionally with time and inversely with Set Size. In addition, a linear system of independent parallel processes with stochastic input sequences would produce a direct relation between Set Size and response time (Lappin, 1978). If $T$ is the process time needed to reach a given response criterion for Set Size $n$, then Set Size $b \bullet n$ should require $b \bullet T$ process time. The hazard rates observed during the motion integration phase satisfy such a proportional relation between Set Size and process time.

After this initial integration phase, however, the rate of awareness, $A(n)$, was not influenced by added motion information, contrary to this normative statistical prediction. Visual awareness and motion integration, $A(n)$ and $M(t)$, were functionally separable, influenced by different parameters, and with no apparent tradeoff between the two processes.

Thus, the rate of visual awareness, $a$, evidently reflects a capacity limit - inconsistent with unlimited-capacity normative models. Statistics of the input information might well influence the rate of visual awareness, but this nature of this influence is not yet known.

Visual search hypotheses Visual search tasks are useful for quantifying the effect of Set Size on rates of search and recognition. Like many other visual search experiments (see Eckstein, 2011; Logan, 2004; Wolfe \& Horowitz, 2004), the present study also found a linear relation between Set Size and mean RT. Such linear functions have often suggested serial processes, but the present results contradict the intuitive concept of a serial search process that progressively reduces the set of not-yet-searched items. Here, the rate of visual awareness was constant over time, independent of the preceding duration of visual process. Other studies have also found that visual search involved such temporal independence, as if the search processes had no memory (e.g., Horowitz \& Wolfe, 1998, 2001). However, if visual process rates - hazard rates - remain constant over time, then a serial/parallel distinction seems insubstantial; and the distinction is not empirical in terms of RTs for detecting a single target in varied Set Sizes (Townsend, 1972, 1976).

An added constraint is that detection rates in this study were always proportional to Set Size - i.e., $h_{1}(t) / h_{2}(t) \approx$ $h_{2}(t) / h_{4}(t) \approx h_{4}(t) / h_{8}(t)$. Thus, the processing time for each observed sample increased in proportion to all the displayed items - regardless of whether the observed samples encompassed only one or many objects at a time, and regardless of whether these samples were processed sequentially or concurrently.

Visual search studies have also suggested sometimes that visual attention operates to select and integrate visual features supplied by “pre-attentive” vision (e.g., E. Palmer et al., 2011;
Treisman \& Gelade, 1980; Wolfe et al, 1989). Pre-attentive vision is sometimes conceived as an unlimited capacity parallel process. And capacity limitations are thought to influence a second stage of selective attention. In the present study, however, two functionally independent component processes were concurrent. The rate of visual awareness was constant throughout, the same both during and after initial motion integration.

How does attention affect visual awareness and resolution? Are levels of visual awareness binary (aware/unaware) or continuously variable? Were observers equally aware at any given time of all the observed objects, or was visual awareness unevenly distributed over the set of objects with greater awareness of an attended subset? The experimental methods and results of this study do not identify variations in levels of awareness nor how such variations might be distributed over time, space, or objects. The direct proportionality of Set Size and detection times, $1 / h_{n}(t)$, is consistent with the hypothesis that divided attention reduced resolution equally for all observed objects, but the empirical results do not reject several alternative hypotheses.

Regardless of how divided attention may have actually reduced visual resolution of objects and motions in this study, the practical effect was as if resolution was divided in proportion to the spread of attention. Indeed, multiple lines of theoretical and experimental research indicate that attention does affect the resolution and/or processing rates for input signals. The following are noteworthy examples.

1) The limited-capacity parallel-process models developed by Townsend and colleagues (e.g., Eidels et al., 2015; Townsend \& Ashby, 1983; Townsend \& Eidels, 2011; Townsend \& Nozawa, 1995; Townsend \& Wenger, $2004 a, b)$ are directly supported by the present results. Wenger \& Gibson (2004) found that attentional pre-cues produced a roughly one-third increase in hazard rates for targets in multi-element displays.

2) The zoom-lens model of Eriksen and colleagues (Eriksen \& Hoffman, 1974; Eriksen \& St. James, 1986; Eriksen \& Yeh, 1985) postulates that resolving power is inversely correlated with the area of focus. Pre-cues to target location produced faster RTs for discriminating target forms and reduced interference from neighboring forms.

3) Bundesen's (1990) "theory of visual attention" (TVA) and expanded neural theory (NTVA) represents the acquisition rate and resolution of visual information as a fixedcapacity parallel race among independent visual signals (e.g., Bundesen, 1990; Bundesen et al., 2005, 2011; Logan, 2002, 2004; Shibuya \& Bundesen, 1988).

4) Neurophysiological normalization: Suppressive feedback from surrounding population activity has been found to reduce firing rates of individual neurons; and this model 
has been used recently to describe effects of attention on both cortical neural responses and behavioral discriminations (e.g., Carandini \& Heeger, 2012; Reynolds \& Desimone, 2003; Reynolds \& Heeger, 2009; Reynolds et al., 2000).

5) Contrast resolution: Pre-cueing can enhance contrast resolution in various tasks requiring detection, localization, and orientation discrimination (Carrasco et al., 2000). Covert attention elicited by a pre-cue increased subjective contrast (Carrasco et al., 2004). Voluntary attention altered perceived relative contrasts of superimposed colors moving in opposite directions (Blaser et al., 1999).

6) Spatial resolution: Yeshurun and Carrasco (1999) found that spatial pre-cuing improved both speed and accuracy in spatial acuity tasks.

7) Spatiotemporal resolution: Koenderink (2012) and Koenderink et al. (2012) demonstrated that attention to meaningful structure reduced perception of motions produced by perturbing spatial and temporal positions of image elements in movies.

The present results are at least qualitatively consistent with the preceding theories and results. The reduced detection rates found here, however, were larger and simpler than in most of these previous studies. The inverse proportionality of hazard rates and Set Size involves no additional free parameters beyond the rate capacity of visual awareness.

A generalized channel capacity of visual awareness? A constant rate-capacity of visual awareness was the primary determinant of target detection in this study. A single rate parameter was invariant with Set Sizes, response times, and motion durations. This invariance is striking, but much remains to be learned about the functions of awareness in complex task environments. Is this a visual capacity, for example, or is it a general multi-modal and cross-modal capacity limit that affects multiple sensory modalities at the same time? Divided attention certainly affects the auditory modality (e.g., Cherry, 1953; Dehais et al., 2014), reducing detection of even noxious multi-modal stimuli (Wayand, Levin, \& Varakin, 2005). The hypothesis that cognitive awareness of virtually all forms of sensory information might be limited by a single channel capacity certainly awaits clarification by a larger research program. And this issue certainly cannot be resolved by a single experiment. The definition and measurement of diverse forms of 'information' entails difficult theoretical problems.

The finding in Experiment 2 that Motion Speed had a substantial effect on the rate of awareness demands further investigation. Importantly, the effects of Motion Speed were both reduced and opposite when the rate of target detection is expressed in spatial units, as bits ${ }^{\circ}$. Thus, the rate of visual awareness evidently entails both spatial and temporal dimensions of motion. Do similar spatial capacity limits apply to the visual awareness of stationary object properties? The answer is not now available.

Analogous questions concern potential effects of the output responses. Invariance of the rate parameter $(a)$ with RT suggests that this capacity limit might not be specific to the response system, but the present evidence is limited. Applications to measures of accuracy and speed/accuracy tradeoff are of particular interest.

An incidental finding was that the rate of awareness was higher in Experiment 1 than in Experiments 2 and 3 for the same Motion Speed. The possibility that differences in temporal uncertainty yield differences in the rate of awareness demands further investigation.

\section{General conclusion}

A constant rate of awareness was a primary determinant of the rate of target detection when attention was divided among multiple moving objects. Visual awareness operated in parallel, independently and concurrently, with visual processes that integrated spatiotemporal information about object motions. The role of visual awareness and its constant capacity were revealed by the hazard rates of target detection.

Acknowledgments This research was supported in part by Phase I of a Small Business Technology Transfer contract on "Information salience" (N00014-12-M-0396) from the Office of Naval Research to Discerning Technologies LLC (DiscernTek), with a subcontract to Vanderbilt University. The authors are grateful to John Moniz and Capt. Benjamin Pimentel of ONR for supervision. We also gratefully recognize the suggestions and help of Dr. R. Michael Templeton (CEO, DiscernTek) through multiple phases of the project. A report of this study was also presented at the annual meeting of the Vision Sciences Society, St. Pete Beach FL, May 2015. The authors are grateful to Dr. Kevin Dieter of Vanderbilt for extensive contributions as an observer and discussions. This report has also benefitted from discussions with Profs. Gordon Logan, James Townsend, Mike Wenger, Philip Smith, and Daniel Levin.

\section{Appendix 1: Evaluating temporal processes by integrated hazard functions and by momentary hazard rates}

This Appendix clarifies the rationale for evaluating relative performance in different conditions and over time by momentary hazard rates, $h(t)$ - rather than by the integrated functions, $H(t)$. Townsend and colleagues have developed a theoretical framework for experimental methods (e.g., Eidels et al, 2015; Townsend \& Nozawa, 1995; Townsend \& Wenger, 2004a, b), statistical methods (Houpt \& Townsend, 2012; Wenger \& Gibson, 2004), and empirical support (Godwin et al., 2015; Wenger \& Gibson, 2004) for evaluating efficiency and capacity by ratios of the integrated hazard functions for 
different conditions. In the present study, however, relative performance was evaluated by ratios of the momentary hazard rates, $h(t)$ - estimated here by slopes of the integrated functions.

Naturally, the two measures have much in common. Both measures are functions of the rank-ordered RTs, independent of the trial sequence, and independent of events in preceding trials; both evaluate survival functions, the numbers of responses that have not yet occurred at a given time; both are ratio scales of performance at a given time; and both trace the time course of a temporal process underlying observed RTs.

Nevertheless, integrated and momentary hazard functions measure different properties of the temporal process. Specifically, suppose that $H_{A}(t), h_{A}(t), H_{B}(t)$, and $h_{B}(t)$ are the integrated and momentary hazard rates at a given time $t$ for two different experimental conditions, $A$ and $B$. Then $H_{A}(t) / H_{B}(t)=y$ does not imply that $h_{A}(t) /$ $h_{B}(t) \approx y$.

The truth of this proposition follows immediately from the fact that the integrated and momentary hazard functions are defined on different sets of RTs: The integrated function is determined by the relative frequency of responses in the preceding RT interval $(0, t)$, but the momentary hazard rate $h(t)$ is estimated by the relative frequency of responses in a following interval $(t, t+\Delta t)$.

Houpt and Townsend (2012) develop statistical methods for estimating and comparing integrated hazard functions for sets of discrete trials. If the hazard function is estimated from a total of $N$ response times, and if $M$ is the number of survivors at time $t$, then an unbiased estimate of the integrated function $H(t)$ is a sum of the hazard rates for each individual response preceding time $t$ :

$$
H(t)=-\ln S(t) \approx[1 / N+1 /(N-1)+\ldots+1 /(M+1)] .
$$

By the same "Nelson-Aalen" procedure (see Houpt \& Townsend, 2012), the momentary hazard rate for an interval of $\Delta t$ beginning at time $t$ containing $Q$ responses is estimated from a similar sum of a succeeding subset of responses:

$$
h(t)=[\ln S(t)-\ln S(t+\Delta t)] \Delta t \approx\left[\begin{array}{c}
1 / M+1 /(M-1) \\
+\ldots+1 /(M-Q+1)
\end{array}\right] / \Delta t .
$$

Empirically, these two computational procedures produced different descriptions of relative performance in different conditions in the present study. Fig. 8 plots the relative values of integrated hazard functions, $\log _{2} H_{n}(t)-\log _{2} H_{1, \text { est. }}(t)$. The four Set Size conditions, $n=\{2,4,6,8\}$, are each compared with

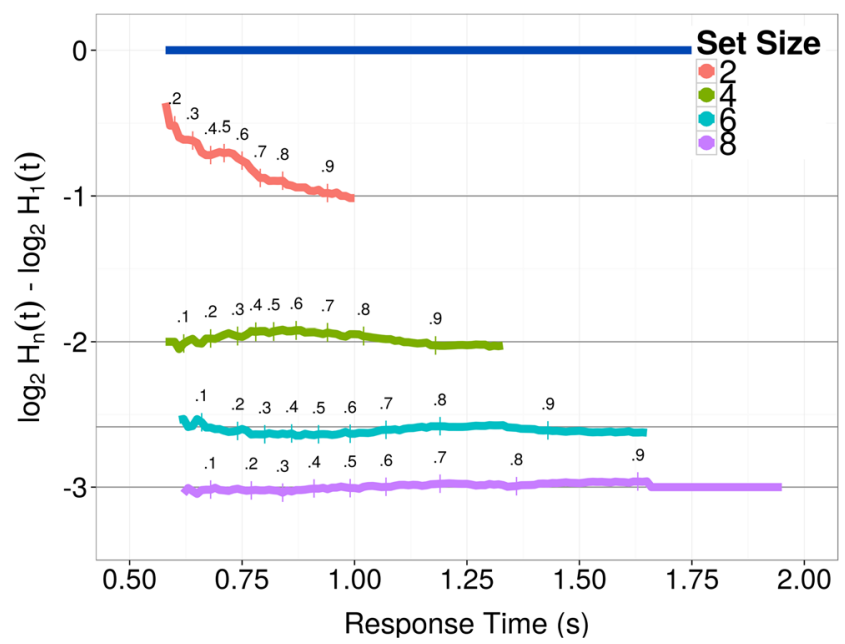

Fig. $8 \log _{2}$ of ratios of integrated hazard functions, $H_{n}(t)$, for each Set Size condition in Exp. 1. Data in the three lower curves, for $n=4,6$, and 8 , were averaged to estimate the baseline for a hypothetical $n=1$ condition. Each curve describes the distribution function for RTs from the 5 th to 95 th percentile; and the deciles from 10th to 90th are marked for each condition. (The curve for Set Size 2 begins at the 16th percentile.) Compare with Fig. 3b, based on the same data

an estimated baseline performance for Set Size $n=1$, where this baseline was estimated by averaging, in each successive $10 \mathrm{~ms}$ bin, values of $n \bullet H_{n}(t)$ for the conditions with $n=4,6$, and 8. (That is, the estimated value of $H_{1, \text { est. }}(t)=\left[4 \cdot H_{4}(t)+\right.$ $\left.6 \cdot H_{6}(t)+8 \cdot H_{8}(t)\right] / 3$ for those bins in which $0.05 \leq F_{n}(t) \leq 0.95$ for all three of these Set Size conditions. When the distribution function at a given time $t$ for one or two of these three Set Size conditions was not within the 5th-95th percentiles, then the baseline was computed by averaging values from the others.)

Before making this graph, we compared the $\log _{2} H_{n}(t)$ values for all six pairs of the four Set Size Conditions. Those comparisons demonstrated what is seen in Fig. 8: The values of $n \bullet H_{n}(t)$ for the Set Size 2 condition were initially greater than those for $n=4,6$, or 8 , while relative values for the latter conditions were approximately proportional to their respective Set Sizes: $H_{i}(t) / H_{j}(t) \approx(i / j)$, for Set Sizes, $i, j=\{4,6,8\}$. Thus, to show these relations among the several Set Size condition, the baseline for the hypothetical $n=1$ condition was estimated from the three larger Set Sizes. For Set Sizes 4, 6, and 8, the relationship between Set Size and hazard function was similar to that in Fig. $3 b$, where $h_{i}(t) / h_{j}(t) \approx(i / j)$. For Set Size 2, however, Fig. 8, does not correspond to Fig. 3b.

Figure 9 shows relationships among the momentary hazard rates. Figure 9 is similar to Fig. $3 b$ except that hazard rates are estimated at successive $5 \%$ quantiles for the 5-75th quantiles, using a sliding $20 \%$ window to compute the hazard rate for the time interval beginning at each successive $5 \%$ increase in the quantile. And the Nelson-Aalen procedure was used to estimate the 


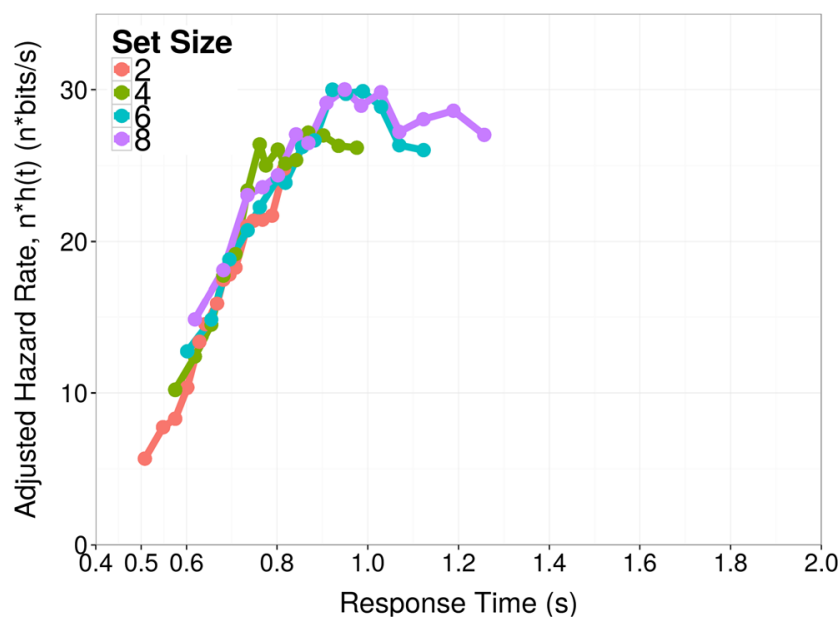

Fig. 9 Momentary hazard rates, $n \bullet h_{n}(t)$, for the data in Fig. 8. Data points are plotted at succeeding 5 percentiles, using a running window of $20 \%$, for $0.05 \leq F_{n}(t) \leq 0.95$. These values were derived from integrated functions estimated by the Nelson-Aalen method (Houpt \& Townsend, 2012)

integrated functions from which these differentials were computed. The RT distributions underlying Figs. 8 and 9 are identical, but these graphs yield different descriptions of relative performance in different conditions and at different times. Figure 8 indicates that the relationship between Set Size and detection performance was relatively better for Set Size 2 than for the three larger Set Sizes; and, additionally, that this Set-Size effect varied with time. Figure 9, however, shows quantitatively consistent and functionally independent effects of both time and Set Size. The descriptive analysis in Fig. 9 is clearer and simpler, as well as more detailed.

The discrepancy between the analyses in Figs. 8 and 9 results from two factors: First, Fig. 8 describes ratios of integrated functions, whereas Fig. 9 describes local rates of change in the integrated functions. Second, as indicated above, the two functions are defined on different subsets of the ordered RTs: The integrated functions in Fig. 8 evaluate the set of RTs $\leq t$, whereas Fig. 9 is based on a subset of slower RTs, $t<R T \leq t+\Delta t$.

Would the integrated functions and momentary rates be similar if both evaluated RTs $\leq t$ ? The momentary hazard rate could be evaluated at the upper bounds of each time interval, rather than at the lower temporal bounds as in Fig. 9 and elsewhere in this paper. Suppose this difference function located at the upper-time-bound is identified as $g\left(t_{2}\right)=\left[H\left(t_{2}\right)-\right.$ $\left.H\left(t_{1}\right)\right] /\left(t_{2}-t_{1}\right)$. The measures of detection rate given by this upper-bound function $g\left(t_{2}\right)$ are identical to those of the lowerbound function $h\left(t_{1}\right)$, but they are evaluated at different times. The integrated function $H(t)$ may also be considered as an integral of the $g\left(t_{2}\right)$ function, with both functions based on RTs $\leq t$. Graphically, data points for the $g\left(t_{2}\right)$ and $h\left(t_{1}\right)$ functions have the same vertical axis coordinates, but the $g\left(t_{2}\right)$ data

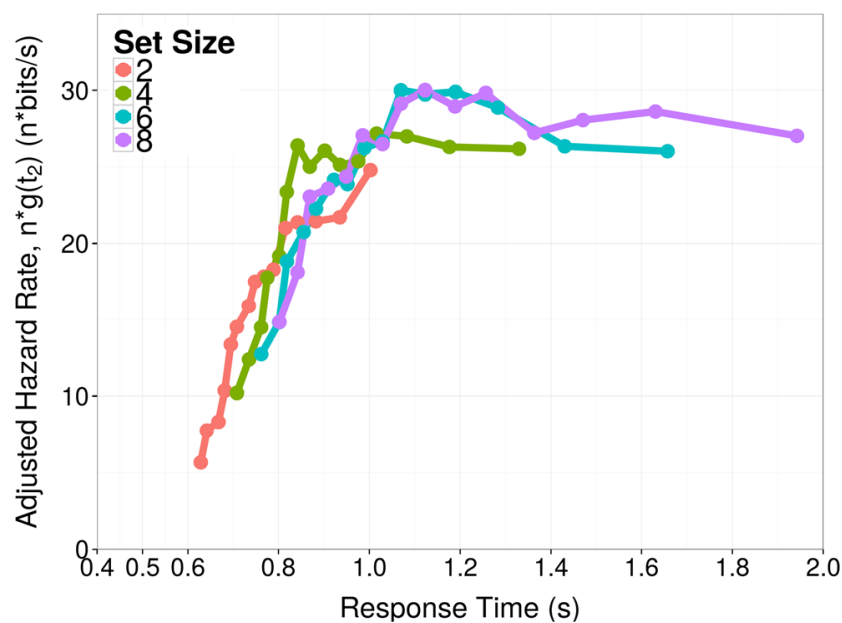

Fig. 10 Hazard rates plotted at the upper bounds of time intervals. Hazard rates on the vertical axis are the same as in Fig. 9, but the time coordinates on the horizontal axis are different. The error variance is almost four times larger in Fig. 10 than Fig. 9 (See text)

points are shifted to the right on the time axis relative to $h\left(t_{l}\right)$; and the temporal shift increases nonlinearly with time. A graph of these $g\left(t_{2}\right)$ functions, Fig. 10, has the same format as Fig. 9, but with data points plotted at the 25-95th percentiles in $5 \%$ increments. Figure 10 resembles Fig. 9, but the relative effects of Set Size and time differ especially for the faster RTs influenced by the Motion Integration process. Consistent with Fig. 8, Fig. 10 displays detection rates for Set Size 2 relatively higher than for the other conditions. Relative performance for Set Size 4 is also higher than for Set Sizes 6 and 8. The adjusted detection rate functions, $n \bullet g\left(t_{2}\right)$, vary among the four Set Size conditions more in Fig. 10 than in Fig. 9. A similar three-parameter model described $91.8 \%$ of the variance in the "upper-bound" hazard rates, $g\left(t_{2}\right)$, in Fig. 10, as compared to $97.8 \%$ of the data in Fig. 9. [The fitting method was the same as for the data in Fig. 3b. The three-parameter model was fit to 16 independent data points, four values of $h\left(t_{1}\right)$ or $g\left(t_{2}\right)$ for each of the four Set Size conditions - at the 10th, 30th, 50th, and 70th percentiles for the $h\left(t_{l}\right)$ functions, and 30th, 50th, 70th, and 90th percentiles for $g\left(t_{2}\right)$. The hazard rates were not multiplied by $n$.]

The conclusion indicated by these analyses is that the momentary hazard rates, $h(t)$ provide a more consistent, more lawful, and simpler description of the temporal process of target detection than descriptive analyses of the integrated hazard functions, $H(t)$.

\section{Appendix 2: Parameter estimation for Experiment 2}

The three parameters of the equations for results of Experiments 1 and 2 are not independent. To evaluate the 
effects of Motion Speed independently for the two component processes, visual awareness and motion integration, a fourstep method was applied to the data for each observer as well as the combined-observer data in Fig. 5:

1) Adjusted hazard rates, $n \bullet h_{n}(t)$, were computed and graphed for each of the nine conditions and each of the four observers - yielding 12 graphs like the three in Fig. 5. These graphs provide visual guidance and a check on the parameter estimation methods.

2) Next, the asymptotic detection rate parameter, $a$, was estimated by averaging adjusted hazard rates, $n \bullet h_{n}(t)$, for data points with the longest RTs for which the hazard rates were uncorrelated with time. These data points were selected by comparing the slope of the regression line between RT and $n \bullet h_{n}(t)$ for successively larger sets of points, beginning with the two longest RTs and including successively more points with faster RTs. Parameter $a$ was then estimated by the mean value of $n \bullet h_{n}(t)$ in the set for which the absolute value of the slope was smallest. This comparative procedure yielded three to seven data points on the right side of the space of $h(t)$ versus RT. These data points were primarily from Set Size 12 and none from Set Size 1. This method insured that the parameters for visual awareness and motion integration were estimated from different sets of data.

3) Using this estimate of parameter $a$, we then estimated the motion integration parameters $c$ and $k$, minimizing the standardized deviation measures, $(O-P)^{2} / P$, with $O$ and $P$ the obtained and predicted hazard rates, as in Experiment 1. Because estimates of parameter $c$, for each observer, varied only slightly and unsystematically between Motion Speed conditions, we used the average of these three estimates of $c$ for all three Motion Speed conditions for each observer.

4) Finally, we estimated parameter $k$ - where the two linear segments intersect - by the value that minimized the squared deviations between obtained and predicted values. Thus, the estimated duration of motion integration is determined solely by parameter $k$. Results are given in Fig. 5 for the combined observers and in Table 3 for the individual observers.

\section{References}

Alvarez, G. A., \& Franconeri, S. L. (2007). How many objects can you track? Evidence for a resource limited attentive tracking mechanism. Journal of Vision, 7(13), 14. 1-10.

Averbach, E., \& Coriell, A. S. (1961). Short-term memory in vision. Bell System Technical Journal, 40, 309-328.
Blaser, E., Sperling, G., \& Lu, Z.-L. (1999). Measuring the amplification of attention. Proceedings of the National Academy of Science, 96(September), 11681-11686.

Bloxom, B. (1984). Estimating response time hazard functions: An exposition and extension. Journal of Mathematical Psychology, 28, 401-420.

Broadbent, D. E. (1958). Perception and communication. London: Pergamon.

Bundesen, C. (1990). A theory of visual attention. Psychological Review, 97(4), 523-547.

Bundesen, C., Habekost, T., \& Kyllingsbaek, S. (2005). A neural theory of visual attention: Bridging cognition and neurophysiology. Psychological Review, 112(2), 291-328.

Bundesen, C., Habekost, T., \& Kyllingsbaek, S. (2011). A neural theory of visual attention and short-term memory (NTVA). Neuropsychologia, 49, 1146-1457.

Carrasco, M., Ling, S., \& Read, S. (2004). Attention alters appearance. Nature Neuroscience, 7(3), 308-313.

Carrasco, M., Penpeci-Talgar, C., \& Eckstein, M. (2000). Spatial covert attention increases contrast sensitivity across the CSF: support for signal enhancement. Vision Research, 40, 1203-1215.

Cechile, R. A. (2003). Mathematical tools for hazard function analysis. Journal of Mathematical Psychology, 47, 478-494.

Chabris, C., \& Simons, D. (2009). The invisible gorilla. New York: Broadway Paperbacks, Crown Publishing Group.

Cherry, E. C. (1953). Some experiments on the recognition of speech with one and two ears. Journal of the Acoustical Society of America, 25, 975-979.

Dehais, F., Causse, M., Vachon, F., Regis, N., Menant, E., \& Tremblay, S. (2014). Failure to detect critical auditory alerts in the cockpit: Evidence for inattentional deafness. Human Factors, 56(4), 631644.

Drew, T., Vo, M. L.-H., \& Wolfe, J. M. (2013). The invisible gorilla strikes again: Sustained inattentional blindness in expert witnesses. Psychological Science, 24(9), 1848-1853.

Eckstein, M. P. (2011). Visual search: A retrospective. Journal of Vision, 11(5), 14. 1-36.

Eidels, A., Townsend, J. T., Hughes, H. C., \& Perry, L. A. (2015). Evaluating perceptual integration: uniting response-time and accuracy-based methodologies. Attention, Perception, \& Psychophysics, 77, 659-680.

Eriksen, C. W., \& Hoffman, J. E. (1974). Selective attention: Noise suppression or signal enhancement? Bulletin of the Psychonomic Society, 4(6), 587-589.

Eriksen, C. W., \& Johnson, H. J. (1964). Storage and decay characteristics of nonattended auditory stimuli. Journal of Experimental Psychology, 68(1), 28-36.

Eriksen, C. W., \& Lappin, J. S. (1967). Selective attention and very shortterm recognition memory for nonsense forms. Journal of Experimental Psychology, 73(3), 358-364.

Eriksen, C. W., \& St. James, J. D. (1986). Visual attention within and around the field of attention: A zoom lens model. Perception \& Psychophysics, 40(4), 225-240.

Eriksen, C. W., \& Yeh, Y.-Y. (1985). Allocation of attention in the visual field. Journal of Experimental Psychology, 11(5), 583-597.

Fehd, H. M., \& Seiffert, A. E. (2010). Looking at the center of the targets helps multiple object tracking. Journal of Vision, 10(4), 19. 1-13.

Fisher, D. L. (1984). Central capacity limits in consistent mapping, visual search tasks: Four channels or more? Cognitive Psychology, 16, 449-484.

Fougnie, D., Asplund, C. L., \& Marois, R. (2010). What are the units of storage in visual working memory? Journal of Vision, 10(12), 27. 1-11.

Franconeri, S. L., Jonathan, S. V., \& Scimeca, J. M. (2010). Tracking multiple objects is limited only by object spacing, not speed, time, or capacity. Psychological Science, 21(7), 920-925. 
Green, D. M., \& Swets, J. A. (1966). Signal detection theory and psychophysics. New York: Wiley.

Godwin, H. J., Houpt, J. W., Walenchok, S. C., Hout, M. C., \& Goldinger, S. D. (2015). Faster than the speed of rejection: Object identification processes during visual search for multiple targets. Journal of Experimental Psychology: Human perception and performance, 41(4), 1007-1020.

Horowitz, T. S., \& Wolfe, J. M. (1998). Visual search has no memory. Nature, 394, 575-577.

Horowitz, T. S., \& Wolfe, J. M. (2001). Search for multiple targets: Remember the targets, forget the search. Perception Psychophysics, 63(2), 272-285.

Houpt, J. W., \& Townsend, J. T. (2012). Statistical measures for workload capacity analysis. Journal of Mathematical Psychology, 56, 341355.

Hyman, R. (1953). Stimulus information as a determinant of reaction time. Journal of Experimental Psychology, 45, 188-196.

Koenderink, J.J. (2012). Chrono-geometry. Utrecht, The Netherlands: De Clootcrans Press. (eBook: http://www.gestaltrevision. be/pdfs/koenderink/ChronoGeometry.pdf)

Koenderink, J.J., van Doorn, A.J., \& Richards, W. (2012). Straight mind - crooked world. European Conference on Visual Perception. Alghero, Sardinia, Italy.

Lappin, J. S. (1978). The relativity of choice behavior and the effect of prior knowledge on the speed and accuracy of recognition. In N. J. Castellan Jr. \& F. Restle (Eds.), Cognitive Theory, Volume 3, Ch. 6 (pp. 139-168). Hillsdale, N.J.: Lawrence Erlbaum.

Lappin, J. S., \& Staller, J. D. (1981). Prior knowledge does not facilitate the perceptual organization of dynamic random-dot patterns. Perception \& Psychophysics, 29(5), 445-456.

Levin, D. T. \& Baker, L. J. (2015). Change blindness and inattentional blindness. In J. Fawcett, E.F. Risko, \& A. Kingstone (Eds.), The Handbook of Attention, Ch. 9 (pp. 199-232). Cambridge, MA: MIT Press.

Logan, G. D. (2002). An instance theory of attention and memory. Psychological Review, 109(2), 376-400.

Logan, G. D. (2004). Cumulative progress in formal theories of visual attention. Annual Reviews of Psychology, 55, 207-234.

Luce, R. D. (1963). Detection and recognition. In R. D. Luce, R. R. Bush, \& E. Galanter (Eds.), Handbook of Mathematical Psychology, Vol. I, Ch. 3 (pp. 103-189). New York: Wiley.

Luce, R. D. (1986). Response times. New York: Oxford University Press.

Luck, S. L., \& Vogel, E. K. (1997). The capacity of visual working memory for features and conjunctions. Nature, 390(20 November), 279-281.

Mack, A. (2003). Inattentional blindness: Looking without seeing. Current directions in psychological science, 12(5), 180-184.

Mack, A., \& Rock, I. (1998). Inattentional blindness. Cambridge, MA: MIT Press.

McCarley, J. S., Vais, M. J., Pringle, H., Kramer, A. F., Irwin, D. E., \& Strayer, D. L. (2004). Conversation disrupts change detection in complex traffic scenes. Human Factors, 46(3), 424-436.

Miller, G. A. (1956). The magical number seven, plus or minus two: Some limits on our capacity for processing information. The Psychological Review, 63(2), 81-97.

Palmer, E. M., Fencsik, D. E., Flusberg, S. J., Horowitz, T. S., \& Wolfe, J. M. (2011). Signal detection evidence for limited capacity in visual search. Attention, Perception, \& Psychophysics, 73(8), 2413-2424.

Peterson, W. W., Birdsall, T. G., \& Fox, W, C. (1954). The theory of signal detectability. IRE Transactions on Information Theory, 4, $171-212$.

Pylyshyn, Z. W., \& Storm, R. W. (1988). Tracking multiple independent targets: Evidence for a parallel tracking mechanism. Spatial Vision, 3(3), 179-197.
Ratcliff, R. (2014). Measuring psychometric functions with the diffusion model. Journal of Experimental Psychology: Human perception and Performance, 40(2), 870-888.

Ratcliff, R., Smith, P. L., Brown, S. D., \& McKoon, G. (2016). Diffusion decision model: Current issues and history. Trends in Cognitive Sciences, 16(4), 260-281.

Reynolds, J. H., \& Desimone, R. (2003). Interacting roles of attention and visual salience in V4. Neuron, 37, 853-863.

Reynolds, J. H., \& Heeger, D. J. (2009). The normalization model of attention. Neuron, 61, 168-185.

Reynolds, J. H., Pasternak, T., \& Desimone, R. (2000). Attention increases sensitivity of V4 neurons. Neuron, 26, 703-714.

Shibuya, H. \& Bundesen, C. (1988). Visual selection from multielement displays: Measuring and modeling effects of exposure duration.

Simons, D.J. (Ed.) (2000). Change blindness and visual memory. East Sussex, UK: Psychology Press. (Also published as a special issue of Visual Cognition, 7, issues 1, 2, \& 3.)

Simons, D. J., \& Chabris, C. F. (1999). Gorillas in our midst: sustained inattentional blindness for dynamic events. Perception, 28, 1059 1074.

Smith, P. L. (2010). From Poisson shot noise to the integrated OrnsteinUhlenbeck process: Neurally principled models of information accumulation in decision-making and response time. Journal of Mathematical Psychology, 54, 266-283.

Smith, P. L., \& Ratcliff, R. (2009). An integrated theory of attention and decision making in visual detection. Psychological Review, 116(2), 283-317.

Sperling, G. (1960). The information available in brief visual presentations. Psychological Monographs: General and Applied, 74(11). Whole No, 498, 1-29.

Stothart, C., Mitchum, A., \& Yehnert, C. (2015). The attentional cost of receiving a cell phone notification. Journal of Experimental Psychology: Human Perception and Performance, 41(4), 893-897.

Strayer, D. L., Drews, F. A., \& Johnston, W. A. (2003). Cell phoneinduced failures of visual attention during simulated driving. Journal of Experimental Psychology: Applied, 9(1), 23-32.

Tanner, W.P., Jr. (1956). Theory of recognition. Journal of the Acoustical Society of America, 28, 882-888. Also in J.A. Swets (Ed.) (1964), Signal detection and recognition by human observers. New York: Wiley.

Townsend, J. T. (1972). Some results concerning the identifiability of parallel and serial processes. British Journal of Mathematical \& Statistical Psychology, 25, 168-199.

Townsend, J. T. (1976). Serial and within-stage independent parallel model equivalence on the minimum completion time. Journal of Mathematical Psychology, 14, 219-238.

Townsend, J. T. (1990). Truth and consequences of ordinal differences in statistical distributions: Toward a theory of hierarchical inference. Psychological Bulletin, 108(3), 551-567.

Townsend, J.T. (1992). On the proper scales for reaction time. In H.-G. Geissler, S.W. Link, \& J.T. Townsend (Eds.), Cognition, Information Processing, and Psychophysics: Basic Issues, Ch. 6, pp. 105-120. Hillsdale, NJ: Lawrence Erlbaum.

Townsend, J. T., \& Ashby, F. G. (1983). Stochastic modeling of elementary psychological processes. Cambridge, UK: Cambridge University Press.

Townsend, J. T., \& Eidels, A. (2011). Workload capacity spaces: A unified methodology for response time measures of efficiency as workload is varied. Psychonomic Bulletin \& Review, 18, 659-681.

Townsend, J. T., \& Nozawa, G. (1995). Spatio-temporal properties of elementary perception: An investigation of parallel, serial, and coactive theories. Journal of Mathematical Psychology, 39, 321-359.

Townsend, J. T., \& Wenger, M. J. (2004a). The serial-parallel dilemma: A case study in a linkage between theory and method. Psychonomic Bulletin \& Review, 11(3), 391-418. 
Townsend, J. T., \& Wenger, M. J. (2004b). A theory of interactive parallel processing: New capacity measures and predictions for a response time inequality series. Psychological Review, 111, 391-418.

Treisman, A. M., \& Gelade, G. (1980). A feature-integration theory of attention. Cognitive Psychology, 12, 97-136.

Usher, M., \& McClelland, J. L. (2004). Loss aversion and inhibition in dynamical models of multialternative choice. Psychological Review, 111(3), 757-769.

Van Zandt, T. (2000). How to fit a response time distribution. Psychonomic Bulletin \& Review, 7(3), 424-465.

Varakin, D. A., Levin, D. T., \& Fidler, R. (2004). Unseen and unaware: Applications of recent research on failures of visual awareness for human-computer interface design. Human-Computer Interaction, 19, 389-421.

Watamaniuk, S. N. J., McKee, S. P., \& Grzywacz, N. M. (1995). Detecting a trajectory embedded in random-direction motion noise. Vision Research, 35(1), 65-77.

Wayand, J. W., Levin, D. T., \& Varakin, D. A. (2005). Inattentional blindness for a noxious multimodal stimulus. American Journal of Psychology, 118, 339-352.
Watson, A. B., \& Turano, K. (1995). The optimal motion stimulus. Vision Research, 35(3), 325-336.

Wenger, M. J., \& Gibson, B. S. (2004). Using hazard functions to assess changes in processing capacity in an attentional cuing paradigm. Journal of Experimental Psychology: Human Perception and Performance, 45, 283-323.

Wolfe, J. M., Cave, K. R., \& Franzel, S. L. (1989). Guided search: An alternative to the feature integration model for visual search. Journal of Experimental Psychology: Human Perception and Performance, 15(3), 419-433.

Wolfe, J. M., \& Horowitz, T. S. (2004). What attributes guide the deployment of visual attention and how do they do it? Nature Reviews. Neuroscience, 5(June), 1-7.

Woodman, G. F., Luck, S. J., \& Schall, J. D. (2007). The role of working memory representations in the control of attention. Cerebral Cortex, 17, 1118-1124.

Yeshurun, Y., \& Carrasco, M. (1999). Spatial attention improves performance in spatial resolution tasks. Vision Research, 39, 293-306. 